\title{
A New Look into the Partial Wave Decomposition of Three-Nucleon Forces
}

\author{
D. Hüber ${ }^{\dagger}$, H. Witała*, A. Nogga ${ }^{\dagger}$, W. Glöckle ${ }^{\dagger}$, H. Kamada ${ }^{\ddagger}$ \\ ${ }^{\dagger}$ Institut für theoretische Physik II, Ruhr-Universität Bochum, D-44780 Bochum, Germany \\ * Institute of Physics, Jagellonian University, PL- 30059 Cracow, Poland \\ $\ddagger$ Paul Scherrer Institut, CH-5232, Villigen PSI, Switzerland
}

(April 10, 2018)

\begin{abstract}
We demonstrate that the partial wave decomposition of three-nucleon forces used up to now in momentum space has to be necessarily unstable at high partial waves. This does not affect the applications performed up to now, which were restricted to low partial waves. We present a new way to perform the partial wave decomposition which is free of that defect. This is exemplified for the most often used $2 \pi$-exchange Tuscon-Melbourne three-nucleon force. For the lower partial waves the results of the old method are reproduced.
\end{abstract}

PACS numbers: 21.30.+y, 21.45.+v, 24.10.-i, 25.10.+s

Typeset using REVTEX 


\section{INTRODUCTION}

Three-nucleon forces (3NF) act for more than two nucleons. The interesting question is their strengths and their signatures.

A first observable where they clearly show up is the binding energy of the triton. Here it is known that the most recent realistic nucleon-nucleon $(\mathrm{NN})$ forces cannot produce the triton binding energy and 3NF's are needed in order to get the experimental number [1] [2].

A next logical step is to look into the $3 \mathrm{~N}$ continuum. Our results based on numerically precise solutions of the three-nucleon (3N) Faddeev equations and realistic NN forces agree overall very well with experimental data [3]. In elastic Nd scattering there is only one discrepancy, which clearly sticks out, the low energy vector analyzing powers, which depend sensitively on the ${ }^{3} P_{j}$ NN force components. There is either an ambiguity in their determination from NN data or one really sees a $3 \mathrm{NF}$ effect. The inclusion of the $3 \mathrm{NF}$ 's, however, which have been worked out up to now, do not diminish that discrepancy [3] [4]. Right now it remains a puzzle [5]. At very low energies, near the $\mathrm{Nd}$ threshold, one has to expect to see 3NF effects, which are linked to the accompanying shift of the triton binding energy. But we predict also scattering observables, which do not scale with the triton binding energy [6] [3]. This is an interesting energy region to be studied experimentally. At higher energies up to about $60 \mathrm{MeV}$ the $3 \mathrm{NF}$ effects we find for cross sections are mostly small (of the order of a few percent) [3] [7] [8] and sometimes even shifting theory in the wrong direction. For certain spin observables there can be larger effects [3]. We apparently stand at the very beginning in understanding 3NF's. At much higher energies, however, above $100 \mathrm{MeV}$ nucleon lab energy, we predict big 3NF effects, though still based on a truncated partial wave basis for the $3 \mathrm{NF}$ [3].

The present article is meant to provide the theoretical and numerical ground to add more partial waves to the 3NF. With the previous algorithm [9] for decomposing the $3 \mathrm{NF}$ into partial waves we ran into unexpected numerical instabilities for higher angular momentum states. 
In section $\llbracket$ we show the origin of this defect using a model 3NF. We demonstrate that the failure in the partial wave decomposition (PWD) used up to now cannot be cured for higher angular momenta.

In section [II] we introduce a new PWD. Thereby we decompose the 3NF into two quasi two-body parts and a recoupling of Jacobi variables in between. This leads to a new PWD, which is free of the inherent difficulties of the old PWD. We perform the new PWD for the $2 \pi$-exchange Tucson-Melbourne (TM) 3NF [10] [9]. Thereby we also comment on the numerics where it is relevant.

We demonstrate in a numerical study in section $\mathbb{\square}$ that for lower partial waves the old and new PWD give the same results for Nd scattering. In a bound state calculation we extend the new PWD to higher partial waves and reproduce exactly the result of the Los Alamos - Iowa group [11].

Finally we summarize in section $\square$.

Appendix A contains the expressions for the permutation operators which are needed in our new approach. They are generalizations of the permutation operators which we usually use [12] [13] in the Faddeev scheme.

The permutation operators lead to interpolation of very many matrix elements. This includes a two-fold interpolation, as is shown in appendix A, for which a very efficient and accurate algorithm has to be used. It turned out, that our usual scheme, namely cubic basis splines [14], requires too much CPU time, which would not allow to calculate the 3NF within a reasonable time. Therefore we use cubic hermitian splines which need much less operations for one interpolation than the basis splines. In appendix B we briefly review the introduction of cubic hermitian splines and rewrite them to the most efficient form for our purposes. 


\section{THE PITFALL IN THE OLD PARTIAL WAVE DECOMPOSITION}

The $2 \pi$-exchange $3 \mathrm{NF}$ is shown in Fig. 1. The three diagrams in Fig. 11 differ only by cyclical and anti-cyclical permutations of the three particles. Therefore it is sufficient to calculate the first diagram and find the full 3NF by the application of proper permutation operators. The first diagram is denoted by $V_{4}^{(1)}$ since particle 1 is singled out.

We need the matrix elements of $V_{4}^{(1)}$ in a basis $|p q \alpha\rangle$, where $p$ and $q$ are Jacobi momenta ( $\vec{p}$ is the relative momentum between particles 2 and 3 and $\vec{q}$ is the momentum of particle 1 relative to the pair 2-3) and $\alpha$ denotes discrete quantum numbers. In detail

$$
|p q \alpha\rangle \equiv\left|p(l s) j q\left(\lambda \frac{1}{2}\right) I(j I) J M ;\left(t \frac{1}{2}\right) T M_{T}\right\rangle
$$

where $l, s$ and $j$ are orbital angular momentum, spin and total angular momentum in the two-body subsystem 2-3. $\lambda, \frac{1}{2}$ and $I$ are the corresponding quantities for particle 1 , and $J M$ denote the conserved total $3 \mathrm{~N}$ angular momentum and its magnetic quantum number. In iso-spin space $t$ refers to particles 2 and 3, which couples with $1 / 2$ to total iso-spin $T$ and its magnetic quantum number $M_{T}$. Thus the matrix elements we are looking for are of the form $\left\langle p q \alpha\left|V_{4}^{(1)}\right| p^{\prime} q^{\prime} \alpha^{\prime}\right\rangle$.

We found that for $p=p^{\prime}$ large, $l=l^{\prime} \geq 4, q=q^{\prime}$ large and $\lambda=\lambda^{\prime} \geq 4$ the matrix elements $\left\langle p q \alpha\left|V_{4}^{(1)}\right| p^{\prime} q^{\prime} \alpha^{\prime}\right\rangle$ are some orders of magnitude larger than neighbouring non symmetric matrix elements. The increase of $p=p^{\prime}, q=q^{\prime}, l=l^{\prime}$ or $\lambda=\lambda^{\prime}$ leads to an explosion in magnitude of the matrix elements. Moreover, we found that in those partial waves the symmetry of $\left\langle p q \alpha\left|V_{4}^{(1)}\right| p^{\prime} q^{\prime} \alpha^{\prime}\right\rangle$ under exchange of the primed and unprimed variables, which is manifest by the operator structure, is violated. This symmetry violation happens even for smaller values of $l$ or $\lambda$.

Such a behaviour hints clearly to a numerical instability of the code. This instability is not caused by an error in the code nor in the formal expressions of the PWD, but by the numerical realisation on a computer which always relies on a finite number of digits. We shall now illustrate the problem for a model using the old PWD. 
The instability is connected to the values of the momenta $p, p^{\prime}, q$ and $q^{\prime}$ and the corresponding angular momentum quantum numbers $l, l^{\prime}, \lambda$ and $\lambda^{\prime}$ and is not related to spin or iso-spin. Thus we can use a spin and iso-spin independent 3NF model for our purpose. Moreover, the behaviour in $q$ and $\lambda$ turns out to be analogical to $p$ and $l$ and therefore it suffices to treat only the $p$ and $l$ dependence in the 3NF model. (The old PWD for the full TM 3NF, which we used up to now, can be found in [9] and in more detail in [15.)

Let us consider $V_{4}^{(1)}$ as depicted in Fig. 2. Neglecting spin and iso-spin, meson-nucleon form factors and replacing the shaded blop by a constant, we are simply left with the two pion propagators

$$
V_{4}^{(1)} \Rightarrow \frac{1}{\vec{Q}^{2}+m_{\pi}^{2}} \frac{1}{{\overrightarrow{Q^{\prime}}}^{2}+m_{\pi}^{2}}
$$

where $\vec{Q}$ and $\vec{Q}^{\prime}$ are given by

$$
\begin{aligned}
\vec{Q} & =\vec{p}-\overrightarrow{p^{\prime}}-\frac{1}{2}\left(\vec{q}-\overrightarrow{q^{\prime}}\right) \\
\overrightarrow{Q^{\prime}} & =\vec{p}-\overrightarrow{p^{\prime}}+\frac{1}{2}\left(\vec{q}-\overrightarrow{q^{\prime}}\right)
\end{aligned}
$$

We further simplify by freezing the $\vec{q}$-dependence and taking only one of the two propagators. Since the problems occur for $p=p^{\prime}$ we choose $|\vec{p}|=\left|\overrightarrow{p^{\prime}}\right|$ and allow only for an angular variation. Thus the model $3 \mathrm{NF}$ becomes

$$
V_{4}^{\text {model }}=\frac{1}{\left(p\left(\hat{p}-\hat{p}^{\prime}\right)+\vec{a}\right)^{2}+m_{\pi}^{2}}
$$

where $\vec{a}$ is a fixed vector.

We define the two angles

$$
x_{2} \equiv \hat{p} \cdot \hat{p^{\prime}} ; \quad x_{1} \equiv\left(\widehat{\vec{p}-\overrightarrow{p^{\prime}}}\right) \cdot \hat{a}
$$

and rewrite eq. (四) as

$$
\begin{aligned}
V_{4}^{\text {model }} & =\frac{1}{2 p^{2}\left(1-x_{2}\right)+a^{2}+2 a p \sqrt{2\left(1-x_{2}\right)} x_{1}+m_{\pi}^{2}} \\
& \equiv \frac{1}{B+A x_{1}}=\frac{A^{-1}}{\frac{B}{A}+x_{1}}
\end{aligned}
$$


with

$$
\begin{aligned}
& A=2 a p \sqrt{2\left(1-x_{2}\right)} \\
& B=2 p^{2}\left(1-x_{2}\right)+a^{2}+m_{\pi}^{2}
\end{aligned}
$$

The expression in eq. (6) is expanded in Legendre polynomials resulting in

$$
V_{4}^{\text {model }}=A^{-1} \sum_{l_{1}=0}^{\infty} \hat{l}_{1} P_{l_{1}}\left(x_{1}\right) Q_{l_{1}}\left(\frac{B}{A}\right)
$$

Here and in the following we use the abbreviation

$$
\hat{l} \equiv 2 l+1
$$

for orbital and spin angular momenta.

The Legendre polynomials themselves can be rewritten in terms of coupled spherical harmonics with respect to the two directions involved in $x_{1}$ :

$$
P_{l_{1}}\left(\left(\overrightarrow{\vec{p}-\overrightarrow{p^{\prime}}}\right) \cdot \hat{a}\right)=\frac{4 \pi}{\sqrt{\hat{l}_{1}}}(-)^{l_{1}} y_{l_{1} l_{1}}^{00}\left(\widehat{\vec{p}-\overrightarrow{p^{\prime}}}, \hat{a}\right)
$$

with

$$
Y_{l_{1} l_{2}}^{l m}(\hat{a}, \hat{b}) \equiv \sum_{\nu} C\left(l_{1} \nu l_{2} m-\nu, l m\right) Y_{l_{1}}^{\nu}(\hat{a}) Y_{l_{2}}^{m-\nu}(\hat{b})
$$

Further one has

$$
Y_{l}^{m}(\widehat{\vec{a}+\vec{b}})=\sum_{\lambda_{1}+\lambda_{2}=l} \frac{a^{\lambda_{1}} b^{\lambda_{2}}}{|\vec{a}+\vec{b}|^{l}} \sqrt{\frac{4 \pi \hat{l} !}{\hat{\lambda}_{1} ! \hat{\lambda}_{2} !}} y_{\lambda_{1} \lambda_{2}}^{l m}(\hat{a}, \hat{b})
$$

We insert now eq. (10) and eq. (12) into eq. (8) and get

$$
\begin{aligned}
V_{4}^{\text {model }} & =A^{-1} \sum_{l_{1}=0}^{\infty} \hat{l}_{1} Q_{l_{1}}\left(\frac{B}{A}\right) \frac{4 \pi}{\sqrt{\hat{l}_{1}}}(-)^{l_{1}} \\
& \times \sum_{\lambda_{1}+\lambda_{2}=l_{1}} \frac{p^{\lambda_{1}}\left(-p^{\prime}\right)^{\lambda_{2}}}{\left|\vec{p}-\overrightarrow{p^{\prime}}\right|^{l_{1}}} \sqrt{\frac{4 \pi \hat{l}_{1} !}{\hat{\lambda}_{1} ! \hat{\lambda}_{2} !}}\left\{y_{\lambda_{1} \lambda_{2}}^{l_{1} m}\left(\hat{p}, \hat{p^{\prime}}\right), Y_{l_{1}}(\hat{a})\right\}^{00}
\end{aligned}
$$

Hereby we used the notation

$$
\left\{a_{l_{1}}, b_{l_{2}}\right\}^{l m} \equiv \sum_{\nu} C\left(l_{1} \nu l_{2} m-\nu, l m\right) a_{l_{1}}^{\nu} b_{l_{2}}^{m-\nu}
$$


to describe the coupling of two quantities $a$ and $b$ with angular momenta $l_{1}$ and $l_{2}$ to $l \mathrm{~m}$. Already here we can see the problem with this type of PWD. The expression in eq. (13) seems to have a singularity for $\vec{p}=\overrightarrow{p^{\prime}}$, though the model force is not singular, of course. The singularity in eq. (13) is only apparent and is removed by the sum over $\lambda_{1}+\lambda_{2}$, if that sum can be evaluated accurately. The next step in the PWD is to expand the $x_{2^{-}}$ dependence in terms of Legendre polynomials analogically to what we have done for the $x_{1}$-dependence. The $x_{2}$ dependence occurs in the denominator $\left|\vec{p}-\vec{p}^{\prime}\right|^{l_{1}}$ and in the Legendre polynomial of the second kind, $Q_{l}(B / A)$. Performing that expansion we separate the singular denominator from the sum over $\lambda_{1}+\lambda_{2}$, which has to cancel the singularity. This separation and the necessarily limited accuracy in the numerical evaluation of the sum over complicated geometrical expressions is the reason for the numerical instability as we shall demonstrate now.

Let us now perform the $x_{2}$ expansion:

$$
\begin{aligned}
V_{4}^{\text {model }} & =\frac{1}{2 a p} \sum_{l_{1}=0}^{\infty} 4 \pi \sqrt{\hat{l}_{1}}(-)^{l_{1}} \sum_{\lambda_{1}+\lambda_{2}=l_{1}}(-)^{\lambda_{2}} \sqrt{\frac{4 \pi \hat{l}_{1} !}{\hat{\lambda}_{1} ! \hat{\lambda}_{2} !}}\left\{y_{\lambda_{1} \lambda_{2}}^{l_{1}}\left(\hat{p}, \hat{p^{\prime}}\right), Y_{l_{2}}(\hat{a})\right\}^{0} \\
& \times \sum_{l_{2}=0}^{\infty} 2 \pi \sqrt{\hat{l}_{2}}(-)^{l_{2}} y_{l_{2} l_{2}}^{00}\left(\hat{p}, \hat{p^{\prime}}\right) H_{l_{1} l_{2}}(p, a)
\end{aligned}
$$

The expansion coefficients $H_{l_{1} l_{2}}(p, a)$ are given by

$$
H_{l_{1} l_{2}}(p, a)=\int_{-1}^{+1} d x_{2} P_{l_{2}}\left(x_{2}\right) \frac{Q_{l_{1}}\left(\frac{2 p^{2}\left(1-x_{2}\right)+a^{2}+m_{\pi}^{2}}{2 a p \sqrt{2\left(1-x_{2}\right)}}\right)}{\left(\sqrt{2\left(1-x_{2}\right)}\right)^{l_{1}+1}}
$$

The rest of the PWD is straightforward: one has to recouple the spherical harmonics in eq. (15) in such a way, that $Y_{\lambda_{1}}(\hat{p})$ gets coupled with $Y_{l_{2}}(\hat{p})$ and $Y_{\lambda_{2}}\left(\hat{p^{\prime}}\right)$ with $Y_{l_{2}}\left(\hat{p^{\prime}}\right)$. The intermediate form can be simplified using

$$
y_{l_{1} l_{2}}^{l m}(\hat{a}, \hat{a})=\sqrt{\frac{\hat{l}_{1} \hat{l}_{2}}{4 \pi \hat{l}}} C\left(l_{1} 0 l_{2} 0, l 0\right) Y_{l}^{m}(\hat{a})
$$

Finally projecting onto definite orbital angular momenta one ends up with

$$
\left\langle p l m\left|V_{4}^{\text {model }}\right| p^{\prime} l^{\prime} m^{\prime}\right\rangle=\pi \frac{(-)^{m^{\prime}}}{a p} \sum_{l_{1}} \sqrt{\hat{l}_{1}} \sum_{l_{2}} \sqrt{\hat{l}_{2}} H_{l_{1} l_{2}}(p, a) Y_{l_{1}}^{-m^{\prime}}(\hat{a})
$$




$$
\begin{aligned}
& \times \sum_{\lambda_{1}+\lambda_{2}=l_{1}} \sqrt{\frac{4 \pi \hat{l}_{1 !}}{\hat{\lambda}_{1} ! \hat{\lambda}_{2} !}} \sqrt{\hat{l}_{2} \hat{\lambda}_{1} \hat{\lambda}_{2}}\left\{\begin{array}{ccc}
l_{1} & \lambda_{1} & \lambda_{2} \\
l_{2} & l^{\prime} & l
\end{array}\right\} \\
& \times C\left(l_{2} 0 \lambda_{1} 0, l 0\right) C\left(l_{2} 0 \lambda_{2} 0, l^{\prime} 0\right) \\
& \times C\left(l_{1} m^{\prime} l_{1}-m^{\prime}, 00\right) C\left(l m l^{\prime} m^{\prime}-m, l_{1} m^{\prime}\right)
\end{aligned}
$$

We see from eq. (18) that the $p$-dependence is now shifted into the expansion coefficients $H_{l_{1} l_{2}}(p, a)$. Since the code for the $3 \mathrm{NF}$ failed for large $p$ we regard the limes of $H_{l_{1} l_{2}}(p, a)$ for $p \rightarrow \infty$. Inspection of eq. (16) suggests to introduce as a new integration variable the argument of $Q_{l_{1}}$ :

$$
z\left(x_{2}\right) \equiv \frac{2 p^{2}\left(1-x_{2}\right)+a^{2}+m_{\pi}^{2}}{2 a p \sqrt{2\left(1-x_{2}\right)}}
$$

Because $z\left(x_{2}\right)$ has a minimum at $x_{2}=1-\frac{a^{2}+m_{\pi}^{2}}{2 p^{2}}$ the integral over $z$ splits into two parts. It results

$$
\begin{aligned}
H_{l_{1} l_{2}}(p, a) & =\int_{\frac{4 p^{2}+a^{2}+m_{\pi}^{2}}{4 a p}}^{\frac{\sqrt{a^{2}+m_{\pi}^{2}}}{a}} d z\left(-\frac{2 a^{2} z}{p^{2}}-\frac{a}{p^{2}} \frac{2 a^{2} z^{2}-\left(a^{2}+m_{\pi}^{2}\right)}{\sqrt{a^{2} z^{2}-\left(a^{2}+m_{\pi}^{2}\right)}}\right) \\
& \times P_{l_{2}}\left(1+\frac{a^{2}+m_{\pi}^{2}}{2 p^{2}}-\frac{a^{2} z^{2}}{p^{2}}-\frac{a z}{p^{2}} \sqrt{a^{2} z^{2}-\left(a^{2}+m_{\pi}^{2}\right)}\right) \\
& \times Q_{l_{1}}(z)\left(2\left(-\frac{a^{2}+m_{\pi}^{2}}{2 p^{2}}+\frac{a^{2} z^{2}}{p^{2}}+\frac{a z}{p^{2}} \sqrt{a^{2} z^{2}-\left(a^{2}+m_{\pi}^{2}\right)}\right)\right)^{-\frac{l_{1}+1}{2}} \\
& +\int_{\frac{\sqrt{a^{2}+m_{\pi}^{2}}}{a}}^{\infty} d z\left(-\frac{2 a^{2} z}{p^{2}}+\frac{a}{p^{2}} \frac{2 a^{2} z^{2}-\left(a^{2}+m_{\pi}^{2}\right)}{\sqrt{a^{2} z^{2}-\left(a^{2}+m_{\pi}^{2}\right)}}\right) \\
& \times P_{l_{2}}\left(1+\frac{a^{2}+m_{\pi}^{2}}{2 p^{2}}-\frac{a^{2} z^{2}}{p^{2}}+\frac{a z}{p^{2}} \sqrt{a^{2} z^{2}-\left(a^{2}+m_{\pi}^{2}\right)}\right) \\
& \times Q_{l_{1}}(z)\left(2\left(-\frac{a^{2}+m_{\pi}^{2}}{2 p^{2}}+\frac{a^{2} z^{2}}{p^{2}}-\frac{a z}{p^{2}} \sqrt{a^{2} z^{2}-\left(a^{2}+m_{\pi}^{2}\right)}\right)\right)^{-\frac{l_{1}+1}{2}}
\end{aligned}
$$

Because $Q_{l_{1}}(z)$ decreases like $z^{-l_{1}-1}$ for large $z, z$ is limited to small values and both arguments of $P_{l_{2}}$ go to 1 for $p \rightarrow \infty$. As a consequence $H_{l_{1} l_{2}}(p, a)$ becomes independent of $l_{2}$. We end up with

$$
\lim _{p \rightarrow \infty} H_{l_{1} l_{2}}(p, a) \propto p^{l_{1}-1}
$$


Because the model force decreases for large momenta the increase of $H$ for $p \rightarrow \infty$ has to be canceled by the sums occurring in eq. (18). Indeed this is the case, as we will show now.

If $H$ is independent of $l_{2}$ we can perform the sum over $l_{2}$ analytically:

$$
\begin{aligned}
& \sum_{l_{2}} \hat{l}_{2}\left\{\begin{array}{ccc}
l_{1} & \lambda_{1} & \lambda_{2} \\
l_{2} & l^{\prime} & l
\end{array}\right\} C\left(l_{2} 0 \lambda_{1} 0, l 0\right) C\left(l_{2} 0 \lambda_{2} 0, l^{\prime} 0\right) \\
= & (-)^{\lambda_{2}} \sqrt{\frac{\hat{l}^{\prime}}{\hat{l}_{1}}} C\left(\lambda_{1} 0 \lambda_{2} 0, l_{1} 0\right) C\left(l_{1} 0 l^{\prime} 0, l 0\right)
\end{aligned}
$$

Using this result we can also perform the sum over $\lambda_{1}+\lambda_{2}$ analytically:

$$
\begin{aligned}
& \sum_{\lambda_{1}+\lambda_{2}=l_{1}}(-)^{\lambda_{2}} \sqrt{\frac{4 \pi\left(2 l_{1}\right) !}{\left(2 \lambda_{1}\right) !\left(2 \lambda_{2}\right) !}} C\left(\lambda_{1} 0 \lambda_{2} 0, l_{1} 0\right) \\
= & \sum_{\lambda_{1}+\lambda_{2}=l_{1}}(-)^{\lambda_{2}} \sqrt{\frac{4 \pi\left(2 l_{1}\right) !}{\left(2 \lambda_{1}\right) !\left(2 \lambda_{2}\right) !}} \sqrt{\hat{l}_{1}} \sqrt{\frac{\left(2 l_{1}-2 \lambda_{1}\right) !\left(2 l_{1}-2 \lambda_{2}\right) !}{\left(2 l_{1}+1\right) !}} \frac{l_{1} !}{\left(l_{1}-\lambda_{1}\right) !\left(l_{1}-\lambda_{2}\right) !} \\
= & (-)^{l_{1}} \sqrt{4 \pi} \sum_{\lambda_{1}}(-)^{\lambda_{1}} \frac{l_{1}\left(l_{1}-1\right) \cdots\left(l_{1}-\lambda_{1}\right)}{\lambda_{1} !} \\
= & (-)^{l_{1}} \sqrt{4 \pi} \sum_{\lambda_{1}}(-)^{\lambda_{1}}\left(\begin{array}{c}
l_{1} \\
\lambda_{1}
\end{array}\right)=(-)^{l_{1}} \sqrt{4 \pi}(1-1)^{l_{1}}
\end{aligned}
$$

From this result we can see two things: firstly the term $(1-1)^{l_{1}}$ cancels indeed $p^{l_{1}-1}$. (A somewhat harder inspection shows that only $p^{-2}$ survives, as it should.) But secondly, to achieve this cancellation one has to add up an alternating sum. This causes loss of digits if the consecutive numbers are of similar magnitude. This is the case here. For large $l_{1}$ it will be impossible to get a reasonable result for $(1-1)^{l_{1}}$.

Looking into the corresponding sums in the code for the full TM $3 \mathrm{NF}$ we found, that for diagonal matrix elements with $p \geq 6 \mathrm{fm}^{-1}, l=4, q \geq 12 \mathrm{fm}^{-1}$ and $\lambda \geq 4$ this cancellation occurs with consecutive numbers being of alternating sign and equal within up to 8 digits. Thereby we lost all numerical accuracy. As a consequence the increase $p^{l_{1}-1}$ from the corresponding $H$ could not be compensated and badly wrong results appeared.

It is also clear that an increase of the numerical accuracy cannot solve that problem. Higher numerical accuracy would only shift the problem to higher $l$ and $\lambda$. 


\section{THE NEW PARTIAL WAVE DECOMPOSITION}

In this section we will present a new PWD, which avoids the introduction of artificial singularities. The new PWD leads to a numerically stable code. We perform this PWD for the TM $2 \pi$-exchange $3 \mathrm{NF}$. In the notation from Fig. 2, this force is given by [9]

$$
\begin{aligned}
V_{4}^{(1)} & =\frac{1}{(2 \pi)^{6}} \frac{g^{2}}{4 m_{n}^{2}} \vec{\sigma}_{2} \cdot \overrightarrow{Q^{\prime}} \vec{\sigma}_{3} \cdot \vec{Q} \frac{F^{2}\left(\vec{Q}^{2}\right)}{{\overrightarrow{Q^{2}}+m_{\pi}^{2}}^{2}} \frac{F^{2}\left({\overrightarrow{Q^{\prime}}}^{2}\right)}{\vec{Q}^{2}+m_{\pi}^{2}} \\
& \times\left[\overrightarrow{\tau_{2}} \cdot \overrightarrow{\tau_{3}}\left(a+b \vec{Q} \cdot \overrightarrow{Q^{\prime}}+c\left(\vec{Q}^{2}+{\overrightarrow{Q^{\prime}}}^{2}\right)\right)+d \overrightarrow{\tau_{1}} \cdot \overrightarrow{\tau_{2}} \times \overrightarrow{\tau_{3}} \sigma_{1} \cdot \vec{Q} \times \overrightarrow{Q^{\prime}}\right]
\end{aligned}
$$

The constants $a, b, c$ and $d$ are determined by low energy theorems [10]. The form factors are parametrized as

$$
F\left(\vec{Q}^{2}\right) \equiv \frac{\Lambda^{2}-m_{\pi}^{2}}{\Lambda^{2}+\vec{Q}^{2}}
$$

with the cut-off parameter $\Lambda$.

The Faddeev scheme [13] [12] [16] [17] we are using requires matrix elements $\left\langle p q \alpha\left|V_{4}^{(1)}(1+P)\right| p^{\prime} q^{\prime} \alpha^{\prime}\right\rangle$, where $P$ is the sum of a cyclical and an anti-cyclical permutation of the three nucleons. Henceforth we add a subscript 1,2 or 3 to the basis states $|p q \alpha\rangle$ in order to distinguish the three different choices of Jacobi momenta, which single out either particles 1, 2 or 3 to be the "spectator nucleon". In that notation we can write

$$
\begin{aligned}
& { }_{1}\left\langle p q \alpha\left|V_{4}^{(1)}(1+P)\right| p^{\prime} q^{\prime} \alpha^{\prime}\right\rangle_{1} \\
= & \mathcal{Y}^{\prime \prime}{ }_{1}\left\langle p q \alpha\left|V_{4}^{(1)}\right| p^{\prime \prime} q^{\prime \prime} \alpha^{\prime \prime}\right\rangle_{3}{ }_{3}\left\langle p^{\prime \prime} q^{\prime \prime} \alpha^{\prime \prime}|(1+P)| p^{\prime} q^{\prime} \alpha^{\prime}\right\rangle_{1}
\end{aligned}
$$

We introduced intermediate states of the type 3 , since they single out the pair (12), which participates in one of the two pion exchanges in the operator $V_{4}^{(1)}$. This is the natural basis to evaluate that specific pion exchange. The other pion exchange is in the pair (13), for which the basis $|p q \alpha\rangle_{2}$ is the natural one. This leads us to the basis idea of the new PWD, to split each term of $V_{4}^{(1)}$ into two parts, which are related to the separate one pion exchanges and which are then necessarily linked by a change of coordinates. We call the corresponding two parts $\left.v_{4}^{(1)}\right|_{3}$ and $\left.v_{4}^{(1)}\right|_{2}$, where the subscripts stand again for the spectator 
nucleon. Thus each of the four parts of $V_{4}^{(1)}$ in eq. (25), $v_{4}^{(1)}$ called in the following, can be cast into the form

$$
\begin{aligned}
& { }_{1}\left\langle p q \alpha\left|v_{4}^{(1)}\right| p^{\prime} q^{\prime} \alpha^{\prime}\right\rangle_{3}=y^{\prime \prime} y^{\prime \prime \prime} \underbrace{\prime \prime \prime \prime}{ }_{1}\left\langle p q \alpha \mid p^{\prime \prime} q^{\prime \prime} \alpha^{\prime \prime}\right\rangle_{2} \\
& { }_{2}\left\langle p^{\prime \prime} q^{\prime \prime} \alpha^{\prime \prime}\left|v_{4}^{(1)}\right|_{2} \mid p^{\prime \prime \prime} q^{\prime \prime \prime} \alpha^{\prime \prime \prime}\right\rangle_{2}{ }_{2}\left\langle p^{\prime \prime \prime} q^{\prime \prime \prime} \alpha^{\prime \prime \prime} \mid p^{\prime \prime \prime \prime} q^{\prime \prime \prime \prime} \alpha^{\prime \prime \prime \prime}\right\rangle_{3} \\
& \times_{3}\left\langle p^{\prime \prime \prime \prime} q^{\prime \prime \prime \prime \prime} \alpha^{\prime \prime \prime \prime \prime}\left|v_{4}^{(1)}\right|_{3} \mid p^{\prime} q^{\prime} \alpha^{\prime}\right\rangle_{3}
\end{aligned}
$$

The advantage of this PWD is that the matrix elements ${ }_{2}\left\langle p^{\prime \prime} q^{\prime \prime} \alpha^{\prime \prime}\left|v_{4}^{(1)}\right|_{2} \mid p^{\prime \prime \prime} q^{\prime \prime \prime} \alpha^{\prime \prime \prime}\right\rangle_{2}$ and ${ }_{3}\left\langle p^{\prime \prime \prime \prime} q^{\prime \prime \prime \prime} \alpha^{\prime \prime \prime \prime}\left|v_{4}^{(1)}\right|_{3} \mid p^{\prime} q^{\prime} \alpha^{\prime}\right\rangle_{3}$ are easily evaluated, and neither these matrix elements nor the two changes of basis states introduce numerical instabilities. These changes of basis states are performed by the application of certain permutation operators, which are given in appendix A. Their actual use requires 2-fold interpolations which are performed with the help of cubic hermitian splines (see appendix B).

The basis states $|p q \alpha\rangle_{1}$ in eq. (27) are antisymmetric in the two-body subsystem (23). This is required in the context of the Faddeev equations [13] [12] [16] [17]. The states $|p q \alpha\rangle_{2}$ occurring in eq. (28) do not have that property, since the permutation operators remove that symmetry and therefore one has to sum without restriction over all quantum numbers to a given total angular momentum $J$ and parity. On the other hand the states of type 3 have again to be chosen antisymmetrical in the corresponding two-body subsystem (12). This follows from eq. (27). The permutation operator $(1+P)$ applied onto $\left|p^{\prime} q^{\prime} \alpha^{\prime}\right\rangle_{1}$ in eq. (27) generates a totally antisymmetrical state and consequently the intermediate states ${ }_{3}\left\langle p^{\prime \prime} q^{\prime \prime} \alpha^{\prime \prime}\right|$ have to be antisymmetrical in the subsystem (12). The expression (27) can further be simplified. One has

$$
P_{13} P_{23}|p q \alpha\rangle_{1}=|p q \alpha\rangle_{3}
$$

and

$$
P_{23} P_{13}(1+P) \equiv P_{23} P_{13}\left(1+P_{12} P_{23}+P_{13} P_{23}\right)=1+P
$$

Consequently 


$$
\begin{aligned}
& { }_{1}\left\langle p q \alpha\left|V_{4}^{(1)}(1+P)\right| p^{\prime} q^{\prime} \alpha^{\prime}\right\rangle_{1} \\
= & \mathcal{Y}^{\prime \prime}{ }_{1}\left\langle p q \alpha\left|V_{4}^{(1)}\right| p^{\prime \prime} q^{\prime \prime} \alpha^{\prime \prime}\right\rangle_{3}{ }_{1}\left\langle p^{\prime \prime} q^{\prime \prime} \alpha^{\prime \prime}|(1+P)| p^{\prime} q^{\prime} \alpha^{\prime}\right\rangle_{1}
\end{aligned}
$$

The basis states defined in eq. (11) are a direct product of space-spin states and iso-spin states:

$$
|p q \alpha\rangle \equiv\left|p q \alpha_{J}\right\rangle\left|\alpha_{T}\right\rangle \equiv\left|p(l s) j q\left(\lambda \frac{1}{2}\right) I(j I) J M\right\rangle\left|\left(t \frac{1}{2}\right) T M_{T}\right\rangle
$$

The iso-spin dependence of $v_{4}^{(1)}$ factorizes off and one is therefore naturally lead not to include the iso-spin dependence into the splitting of $v_{4}^{(1)}$ and to evaluate the iso-spin matrix elements ${ }_{2}\left\langle\alpha_{T}^{\prime \prime}|\ldots| \alpha_{T}^{\prime}\right\rangle_{3}$ directly. One easily finds

$$
{ }_{2}\left\langle\left(t \frac{1}{2}\right) T M_{T}\left|\vec{\tau}_{2} \cdot \vec{\tau}_{3}\right|\left(t^{\prime} \frac{1}{2}\right) T^{\prime} M_{T}^{\prime}\right\rangle_{3}=\delta_{T T^{\prime}} \delta_{M_{T} M_{T^{\prime}}}(-6)(-)^{t} \sqrt{\hat{t} \hat{t}}\left\{\begin{array}{ccc}
\frac{1}{2} & \frac{1}{2} & t^{\prime} \\
\frac{1}{2} & 1 & \frac{1}{2} \\
t & \frac{1}{2} & T
\end{array}\right\}
$$

and

$$
\begin{aligned}
& 2\left\langle\left(t \frac{1}{2}\right) T M_{T}\left|\vec{\tau}_{1} \cdot \vec{\tau}_{2} \times \vec{\tau}_{3}\right|\left(t^{\prime} \frac{1}{2}\right) T^{\prime} M_{T}^{\prime}\right\rangle_{3} \\
& =\delta_{T T^{\prime}} \delta_{M_{T} M_{T^{\prime}}} 24 i(-)^{2 T} \sqrt{\hat{t} \hat{t}^{\prime}} \sum_{\lambda=\frac{1}{2}}^{t+\frac{1}{2}}(-)^{3 \lambda+\frac{1}{2}}\left\{\begin{array}{ccc}
\lambda & \frac{1}{2} & 1 \\
\frac{1}{2} & \frac{1}{2} & t
\end{array}\right\}\left\{\begin{array}{ccc}
T & \frac{1}{2} & t \\
\frac{1}{2} & 1 & \lambda \\
t^{\prime} & \frac{1}{2} & \frac{1}{2}
\end{array}\right\}
\end{aligned}
$$

These matrix elements will be denoted by ${ }_{2}\left\langle\alpha_{T}\left|I_{4}^{(1)}\right| \alpha_{T}^{\prime}\right\rangle_{3}$ in the following. Restricting the splitting of $v_{4}^{(1)}$ then to the space-spin parts and calling the two parts $\left.w_{4}^{(1)}\right|_{2}$ and $\left.w_{4}^{(1)}\right|_{3}$ eq. (28) achieves the form

$$
\begin{aligned}
& { }_{1}\left\langle p q \alpha\left|v_{4}^{(1)}\right| p^{\prime} q^{\prime} \alpha^{\prime}\right\rangle_{3}=\mathcal{E}^{\prime \prime} \mathbb{Y}^{1} \mathscr{Y}^{2}{ }_{1}\left\langle p q \alpha \mid p^{\prime \prime} q^{\prime \prime} \alpha^{\prime \prime}\right\rangle_{2} \\
\times & \left({ }_{2}\left\langle p^{\prime \prime} q^{\prime \prime} \alpha_{J}^{\prime \prime}\left|w_{4}^{(1)}\right|_{2} \mid p_{1} q_{1} \alpha_{1 J}\right\rangle_{2}{ }_{2}\left\langle p_{1} q_{1} \alpha_{1 J} \mid p_{2} q_{2} \alpha_{2 J}\right\rangle_{3}\right. \\
\times & \left.{ }_{3}\left\langle p_{2} q_{2} \alpha_{2 J}\left|w_{4}^{(1)}\right|{ }_{3} \mid p^{\prime} q^{\prime} \alpha_{J}^{\prime}\right\rangle_{3}\right) \\
\times & { }_{2}\left\langle\alpha_{T}^{\prime \prime}\left|I_{4}^{(1)}\right| \alpha_{T}^{\prime}\right\rangle_{3}
\end{aligned}
$$

In eq. (35) we introduced intermediate sums $\mathcal{Y}^{1}$ and $\mathcal{Y}^{2}$ where the related quatities carry the indices 1 and 2. Since the quasi two-body forces $\left.w_{4}^{(1)}\right|_{2,3}$ are diagonal in the spectator 
quantum numbers, the intermediate sums over $\alpha_{1 J}$ and $\alpha_{2 J}$ are quite restricted and the main introduction of auxiliary states in eq. (35) are the ones with $\alpha^{\prime \prime}$, which moreover are unconstrained by any symmetry. The $\alpha^{\prime}$ states are anti-symmetrical as mentioned above but otherwise unconstrained due to eq. (31). We should also note that the operators $\left.w_{4}^{(1)}\right|_{2,3}$ can change the parity and the total angular momenta by \pm 1 , as will be shown below.

The evaluation of the permutation operators in eqs. (31) and (35) consumes essentially all the CPU time; the time for the calculation of the $\left.w_{4}^{(1)}\right|_{2,3}$ matrix elements is negligible.

Let us now regard the matrix elements for $\left.w_{4}^{(1)}\right|_{2,3}$ related to the four terms proportional to the constants $a, b, c$ and $d$ in eq. (25). We omit the common factor $\frac{1}{(2 \pi)^{6}} \frac{g^{2}}{4 m_{N}^{2}}$ and the constants $a, b, c$ and $d$.

The a-term naturally splits into two parts, one related to a pion exchange between particles 1 and 3,

$$
\left.w_{4}^{(1)}\right|_{2} ^{a}=F^{2}\left(\vec{Q}^{2}\right) \frac{\vec{\sigma}_{3} \cdot \vec{Q}}{\vec{Q}^{2}+m_{\pi}^{2}}
$$

and the other to a pion exchange between particles 1 and 2,

$$
\left.w_{4}^{(1)}\right|_{3} ^{a}=F^{2}\left({\overrightarrow{Q^{\prime}}}^{2}\right) \frac{\vec{\sigma}_{2} \cdot \overrightarrow{Q^{\prime}}}{{\overrightarrow{Q^{\prime}}}^{2}+m_{\pi}^{2}}
$$

Consequently one has to evaluate

$$
\begin{aligned}
& M_{a}^{J, 2} \equiv{ }_{2}\left\langle p q \alpha_{J}\left|F^{2}\left(\vec{Q}^{2}\right) \frac{\vec{\sigma}_{3} \cdot \vec{Q}}{\vec{Q}^{2}+m_{\pi}^{2}}\right| p_{1} q_{1} \alpha_{1 J}\right\rangle_{2} \\
& M_{a}^{J, 3} \equiv{ }_{3}\left\langle p_{2} q_{2} \alpha_{2 J}\left|F^{2}\left({\overrightarrow{Q^{\prime}}}^{2}\right) \frac{\vec{\sigma}_{2} \cdot \vec{Q}^{\prime}}{{\overrightarrow{Q^{\prime}}}^{2}+m_{\pi}^{2}}\right| p^{\prime} q^{\prime} \alpha_{J}^{\prime}\right\rangle_{3}
\end{aligned}
$$

The states $\alpha_{J}$ from eq. (32) couple the total angular momenta of the two-body subsystem and the third particle:

$$
\left|\alpha_{J}\right\rangle=\left\{\left|\alpha_{j}\right\rangle\left|\alpha_{I}\right\rangle\right\}^{J M} \equiv\left\{|(l s) j\rangle\left|\left(\lambda \frac{1}{2}\right) I\right\rangle\right\}^{J M}
$$

Further the pion momentum $\vec{Q}$ from Fig. 2 is expressed in terms of the Jacobi momenta [13] in the (13) subsystem as 


$$
\vec{Q}=\vec{p}-\vec{p}_{1}
$$

Apparently $M_{a}^{J, 2}$ is diagonal in the quantum numbers of the spectator nucleon, and we find

$$
\begin{aligned}
M_{a}^{J, 2} & =\frac{\delta\left(q-q_{1}\right)}{q^{2}} \delta_{\lambda \lambda_{1}} \delta_{I I_{1}} \sum_{m m_{1}} C(j m I M-m, J M) C\left(j_{1} m_{1} I_{1} M_{1}-m_{1}, J_{1} M_{1}\right) \\
& \times{ }_{2}\left\langle p j m\left|\frac{\vec{\sigma}_{3} \cdot\left(\vec{p}-\vec{p}_{1}\right)}{\left(\vec{p}-\vec{p}_{1}\right)^{2}+m_{\pi}^{2}} F^{2}\left(\left(\vec{p}-\vec{p}_{1}\right)^{2}\right)\right| p_{1} j_{1} m_{1}\right\rangle_{2}
\end{aligned}
$$

The scalar $\vec{\sigma}_{3} \cdot\left(\vec{p}-\vec{p}_{1}\right)$ formed out of two tensor operators of rank 1 can be decomposed using standard techniques [13] as

$$
\begin{aligned}
\vec{\sigma}_{3} \cdot\left(\vec{p}-\vec{p}_{1}\right) & =-\sqrt{3}\left\{\sigma(3),\left(\vec{p}-\vec{p}_{1}\right)_{1}\right\}^{00} \\
& =-4 \pi \sum_{a+b=1} p^{a}\left(-p_{1}\right)^{b}\left\{\sigma(3), y_{a b}^{1}\left(\hat{p}, \hat{p}_{1}\right)\right\}^{00}
\end{aligned}
$$

As in eq. (14) the curly brackets denote angular momentum coupling and $y$ the product of two spherical harmonics coupled to angular momentum 1. Note that in contrast to eq. (13) there arises no inverse power of $\left|\vec{p}-\vec{p}_{1}\right|$, which would lead to an artificial singularity with the fatal numerical consequences described in section $\mathbb{1}$.

Next we expand the pion propagator and form factors into Legendre polynomials depending on $x \equiv \hat{p} \cdot \hat{p}_{1}$. We rewrite

$$
f(x) \equiv \frac{1}{\left(\vec{p}-\vec{p}_{1}\right)^{2}+m_{\pi}^{2}}\left(\frac{\Lambda^{2}-m_{\pi}^{2}}{\Lambda^{2}+\left(\vec{p}-\vec{p}_{1}\right)^{2}}\right)^{2}
$$

as

$$
f(x)=\frac{\left(\Lambda^{2}-m_{\pi}^{2}\right)^{2}}{\left(2 p p_{1}\right)^{3}}\left[\frac{1}{\left(B_{\Lambda}-B_{m_{\pi}}\right)^{2}}\left(\frac{1}{B_{m_{\pi}}-x}-\frac{1}{B_{\Lambda}-x}\right)-\frac{1}{B_{\Lambda}-B_{m_{\pi}}} \frac{1}{\left(B_{\Lambda}-x\right)^{2}}\right]
$$

with

$$
\begin{aligned}
B_{\Lambda} & \equiv \frac{p^{2}+p_{1}^{2}+\Lambda^{2}}{2 p p_{1}} \\
B_{m_{\pi}} & \equiv \frac{p^{2}+p_{1}^{2}+m_{\pi}^{2}}{2 p p_{1}}
\end{aligned}
$$

It results 


$$
f(x)=\sum_{\bar{l}=0}^{\infty} \frac{\hat{\bar{l}}}{2} P_{\bar{l}}(x) H_{\bar{l}}\left(p, p_{1}\right)
$$

with

$$
H_{\bar{l}}\left(p, p_{1}\right)=\frac{1}{p p_{1}}\left(Q_{\bar{l}}\left(B_{m_{\pi}}\right)-Q_{\bar{l}}\left(B_{\Lambda}\right)\right)+\frac{\Lambda^{2}-m_{\pi}^{2}}{2\left(p p_{1}\right)^{2}} Q_{\bar{l}}^{\prime}\left(B_{\Lambda}\right)
$$

These steps lead to the intermediate result for the matrix element in eq. (41)

$$
\begin{aligned}
M_{a}^{j, 2} & \equiv{ }_{2}\left\langle p j m\left|\frac{\vec{\sigma}_{3} \cdot\left(\vec{p}-\vec{p}_{1}\right)}{\left(\vec{p}-\vec{p}_{1}\right)^{2}+m_{\pi}^{2}} F^{2}\left(\left(\vec{p}-\vec{p}_{1}\right)^{2}\right)\right| p_{1} j_{1} m_{1}\right\rangle_{2} \\
& =-\frac{(4 \pi)^{2}}{2}{ }_{2}\langle p j m| \sum_{a+b=1} p^{a}\left(-p_{1}\right)^{b}\left\{\sigma(3), Y_{a b}^{1}\left(\hat{p}, \hat{p}_{1}\right)\right\}^{00} \\
& \times \sum_{\bar{l}=0}^{\infty}(-)^{\bar{l}} \sqrt{\hat{l}} H_{\bar{l}}\left(p, p_{1}\right) Y_{\overline{l l}}^{00}\left(\hat{p}, \hat{p}_{1}\right)\left|p_{1} j_{1} m_{1}\right\rangle_{2}
\end{aligned}
$$

or

$$
\begin{aligned}
M_{a}^{j, 2} & =-2 \pi \sqrt{3} \sum_{a+b=1} p^{a} p_{1}^{b} \sum_{\bar{l}=0}^{\infty} \hat{\bar{l}} H_{\bar{l}}\left(p, p_{1}\right) \sum_{i_{1} i_{2}}(-)^{i_{2}} \\
& \times\left\{\begin{array}{ccc}
a & b & 1 \\
i_{2} & i_{1} & \bar{l}
\end{array}\right\} C\left(a 0 \bar{l} 0, i_{1} 0\right) C\left(b 0 \bar{l} 0, i_{2} 0\right) \\
& \times{ }_{2}\left\langle j m\left|\left\{\sigma(3), Y_{i_{1} i_{2}}^{1}\left(\hat{p}, \hat{p}_{1}\right)\right\}^{00}\right| j_{1} m_{1}\right\rangle_{2}
\end{aligned}
$$

This can be expressed in terms of reduced matrix elements:

$$
\begin{aligned}
{ }_{2}\left\langle j m\left|\left\{\sigma(3), \mathcal{Y}_{i_{1} i_{2}}^{1}\left(\hat{p}, \hat{p}_{1}\right)\right\}^{00}\right| j_{1} m_{1}\right\rangle_{2} & =\delta_{j j_{1}} \delta_{m m_{1}} \frac{\sqrt{\hat{l} \hat{s}}}{\sqrt{3}}(-1)^{1+j+l+s_{1}}\left\{\begin{array}{ccc}
l_{1} & s_{1} & j \\
s & l & 1
\end{array}\right\} \\
& \times{ }_{2}\left\langle l\left\|\mathcal{Y}_{i_{1} i_{2}}^{1}\left(\hat{p}, \hat{p}_{1}\right)\right\| l_{1}\right\rangle_{2}{ }_{2}\left\langle s\|\sigma(3)\| s_{1}\right\rangle_{2}
\end{aligned}
$$

with

$$
{ }_{2}\left\langle s|| \sigma(3) \| s_{1}\right\rangle_{2}=\sqrt{6} \sqrt{\hat{s_{1}}}(-)^{s+1}\left\{\begin{array}{ccc}
\frac{1}{2} & \frac{1}{2} & s_{1} \\
1 & s & \frac{1}{2}
\end{array}\right\}
$$

and

$$
{ }_{2}\left\langle l|| Y_{i_{1} i_{2}}^{L}\left(\hat{p}, \hat{p}_{1}\right)|| l_{1}\right\rangle_{2}=\delta_{i_{1} l} \delta_{i_{2} l_{1}}(-)^{L-l} \sqrt{\frac{\hat{L}}{\hat{l}}}
$$

In view of later use the expression (53) is formulated for general $L$. Note we define the reduced matrix element as 


$$
C\left(L m-m_{1} l_{1} m_{1}, l m\right)\left\langle l|| \mathcal{O}^{L}|| l_{1}\right\rangle \equiv\left\langle l m\left|\mathcal{O}^{L M}\right| l_{1} m_{1}\right\rangle
$$

The result for $M_{a}^{j, 2}$ is

$$
\begin{aligned}
M_{a}^{j, 2} & =6 \pi \sqrt{2} \delta_{j j_{1}} \delta_{m m_{1}}(-)^{j+s+s_{1}+l_{1}} \sqrt{\hat{s} \hat{s}_{1}}\left\{\begin{array}{ccc}
\frac{1}{2} & \frac{1}{2} & s_{1} \\
1 & s & \frac{1}{2}
\end{array}\right\}\left\{\begin{array}{ccc}
l_{1} & s_{1} & j \\
s & l & 1
\end{array}\right\} \\
& \times \sum_{a+b=1} p^{a} p_{1}^{b} \sum_{\bar{l}=0}^{\infty} \hat{\bar{l}} H_{\bar{l}}\left(p, p_{1}\right)\left\{\begin{array}{ccc}
a & b & 1 \\
l_{1} & l & \bar{l}
\end{array}\right\} C(a 0 \bar{l} 0, l 0) C\left(b 0 \bar{l} 0, l_{1} 0\right)
\end{aligned}
$$

Finally we put this expression into eq. (41) for $M_{a}^{J, 2}$. This allows the summation over the magnetic quantum numbers $m$ and $m_{1}$. We also can perform the sums over $a+b=1$ and $\bar{l}$. We end up with

$$
\begin{aligned}
M_{a}^{J, 2} & =\frac{\delta\left(q-q_{1}\right)}{q^{2}} \delta_{j j_{1}} \delta_{\lambda \lambda_{1}} \delta_{I I_{1}} \delta_{J J_{1}} \delta_{M M_{1}} \delta_{\left|l-l_{1}\right|, 1} \\
& \times 2 \pi \sqrt{6}(-)^{j+1+s+s_{1}} \sqrt{\hat{s} \hat{s}_{1}}\left\{\begin{array}{ccc}
\frac{1}{2} & \frac{1}{2} & s_{1} \\
1 & s & \frac{1}{2}
\end{array}\right\}\left\{\begin{array}{ccc}
l_{1} & s_{1} & j \\
s & l & 1
\end{array}\right\} \\
& \times \sqrt{\max \left(l, l_{1}\right)}\left(p H_{l_{1}}\left(p, p_{1}\right)-p_{1} H_{l}\left(p, p_{1}\right)\right)(-)^{\max \left(l, l_{1}\right)}
\end{aligned}
$$

This result for $M_{a}^{J, 2}$ is a very simple expression, in contrast to the $a$-term using the old PWD (see [9] and [15]). The complicated structure of the 3NF in our new PWD is hidden in the three permutation operators of eqs. (31) and (35). The latter ones are, however, well under control algebraically (see appendix A) and numerically. The $M_{a}^{J, 3}$ can be gained out of $M_{a}^{J, 2}$ by carefully replacing quantities with the result

$$
M_{a}^{J, 2}\left(p \alpha, p_{1} \alpha_{1}\right)=(-)^{1+s+s_{1}} M_{a}^{J, 3}\left(p_{1} \alpha_{1}, p \alpha\right)
$$

The splitting of the operator $v_{4}^{(1)}$ introduces parity changes for the individual terms $M_{a}^{J, 2}$ and $M_{a}^{J, 3}$, as is obvious from eq. (56). Their product, however, conserves parity, as it should.

The c-term is very similar to the a-term, only $\vec{Q}^{2}$ and $\vec{Q}^{\prime 2}$ factors occur additionally:

$$
\begin{aligned}
& M_{c}^{J, 2} \equiv{ }_{2}\left\langle p q \alpha_{J}\left|\vec{Q}^{2} F^{2}\left(\vec{Q}^{2}\right) \frac{\vec{\sigma}_{3} \cdot \vec{Q}}{\vec{Q}^{2}+m_{\pi}^{2}}\right| p_{1} q_{1} \alpha_{1 J}\right\rangle_{2} \\
& M_{c}^{J, 3} \equiv{ }_{3}\left\langle p_{2} q_{2} \alpha_{2 J}\left|{\overrightarrow{Q^{\prime}}}^{2} F^{2}\left({\overrightarrow{Q^{\prime}}}^{2}\right) \frac{\vec{\sigma}_{2} \cdot \vec{Q}^{\prime}}{{\overrightarrow{Q^{\prime}}}^{2}+m_{\pi}^{2}}\right| p^{\prime} q^{\prime} \alpha_{J}^{\prime}\right\rangle_{3}
\end{aligned}
$$

This leads to a replacement of $H$ by 


$$
\tilde{H}_{\bar{l}}\left(p, p_{1}\right)=-\frac{m_{\pi}^{2}}{p p_{1}}\left(Q_{\bar{l}}\left(B_{m_{\pi}}\right)-Q_{\bar{l}}\left(B_{\Lambda}\right)\right)-\frac{\Lambda^{2}-m_{\pi}^{2}}{2\left(p p_{1}\right)^{2}} \Lambda^{2} Q_{\bar{l}}^{\prime}\left(B_{\Lambda}\right)
$$

in eq. (56) and provides $M_{c}^{J, 2}$ and $M_{c}^{J, 3}$. The symmetry relation eq. (57) is also valid for $M_{c}^{J, 2}$ and $M_{c}^{J, 3}$.

The b-term in eq. (25) has the scalar product $\vec{Q} \cdot \overrightarrow{Q^{\prime}}$ on top of the structure of the a-term. That scalar product $\vec{Q} \cdot \vec{Q}^{\prime}$ can be rewritten as:

$$
\begin{aligned}
\vec{Q} \cdot \vec{Q}^{\prime} & =-\sqrt{3}\left\{Q_{1}, Q_{1}^{\prime}\right\}^{00}=-\sqrt{3} \frac{4 \pi}{3} Q Q^{\prime} y_{11}^{00}\left(\hat{Q}, \hat{Q}^{\prime}\right) \\
& =\frac{4 \pi}{3} Q Q^{\prime} \sum_{\mu}(-)^{\mu} Y_{1}^{\mu}(\hat{Q}) Y_{1}^{-\mu}\left(\hat{Q}^{\prime}\right)
\end{aligned}
$$

This leads to the amplitudes

$$
\begin{aligned}
& M_{b}^{J, 2} \equiv \sqrt{\frac{4 \pi}{3}}{ }_{2}\left\langle p q \alpha_{J}\left|Q Y_{1}^{\mu}(\hat{Q}) F^{2}\left(\vec{Q}^{2}\right) \frac{\vec{\sigma}_{3} \cdot \vec{Q}}{{\overrightarrow{Q^{2}}}^{2}+m_{\pi}^{2}}\right| p_{1} q_{1} \alpha_{1 J}\right\rangle_{2} \\
& M_{b}^{J, 3} \equiv \sqrt{\frac{4 \pi}{3}}{ }_{3}\left\langle p_{2} q_{2} \alpha_{2 J}\left|Q^{\prime} Y_{1}^{-\mu}\left(\hat{Q}^{\prime}\right) F^{2}\left({\overrightarrow{Q^{\prime}}}^{2}\right) \frac{\vec{\sigma}_{2} \cdot \vec{Q}^{\prime}}{{\overrightarrow{Q^{\prime}}}^{2}+m_{\pi}^{2}}\right| p^{\prime} q^{\prime} \alpha_{J}^{\prime}\right\rangle_{3}
\end{aligned}
$$

Now similarly to eq.(41) one has

$$
\begin{aligned}
M_{b}^{J, 2} & =\frac{\delta\left(q-q_{1}\right)}{q^{2}} \delta_{\lambda \lambda_{1}} \delta_{I I_{1}} \sum_{m m_{1}} C(j m I M-m, J M) C\left(j_{1} m_{1} I_{1} M_{1}-m_{1}, J_{1} M_{1}\right) \\
& \times \sqrt{\frac{4 \pi}{3}}{ }_{2}\left\langle p j m\left|\frac{\vec{\sigma}_{3} \cdot\left(\vec{p}-\vec{p}_{1}\right)}{\left(\vec{p}-\vec{p}_{1}\right)^{2}+m_{\pi}^{2}} F^{2}\left(\left(\vec{p}-\vec{p}_{1}\right)^{2}\right)\right| \vec{p}-\vec{p}_{1}\left|Y_{1}^{\mu}\left(\overrightarrow{p-\vec{p}_{1}}\right)\right| p_{1} j_{1} m_{1}\right\rangle_{2}
\end{aligned}
$$

Its evaluation requires the following recoupling:

$$
\begin{aligned}
& Y_{1}^{\mu}\left(\vec{p}-\vec{p}_{1}\right) \vec{\sigma}(3) \cdot\left(\vec{p}-\vec{p}_{1}\right)=-\sqrt{4 \pi}\left|\vec{p}-\vec{p}_{1}\right|\left\{Y_{1}\left(\overrightarrow{p-\vec{p}_{1}}\right),\left\{\sigma(3), Y_{1}\left(\overrightarrow{p-\vec{p}_{1}}\right)\right\}^{0}\right\}^{1, \mu} \\
= & \frac{\sqrt{4 \pi}}{3}\left|\vec{p}-\vec{p}_{1}\right| \sum_{i_{1}}(-)^{i_{1}+1} \sqrt{\hat{i_{1}}}\left\{\sigma(3), Y_{11}^{i_{1}}\left(\overrightarrow{p-\vec{p}_{1}}, \overrightarrow{p-\vec{p}_{1}}\right)\right\}^{1, \mu} \\
= & \left|\vec{p}-\vec{p}_{1}\right| \sum_{i_{1}}(-)^{i_{1}+1} C\left(1010, i_{1} 0\right)\left\{\sigma(3), Y_{i_{1}}\left(\overrightarrow{p-\vec{p}_{1}}\right)\right\}^{1, \mu} \\
= & \left|\vec{p}-\vec{p}_{1}\right|\left(\frac{1}{\sqrt{3}}\left\{\sigma(3), Y_{0}\left(\overrightarrow{p-\vec{p}_{1}}\right)\right\}^{1, \mu}-\sqrt{\frac{2}{3}}\left\{\sigma(3), Y_{2}\left(\overrightarrow{p-\vec{p}_{1}}\right)\right\}^{1, \mu}\right)
\end{aligned}
$$

As an intermediate step using eq. (12) we get

$$
M_{b}^{j, 2} \equiv \sqrt{\frac{4 \pi}{3}}{ }_{2}\left\langle p j m\left|\frac{\vec{\sigma}_{3} \cdot\left(\vec{p}-\vec{p}_{1}\right)}{\left(\vec{p}-\vec{p}_{1}\right)^{2}+m_{\pi}^{2}} F^{2}\left(\vec{p}-\vec{p}_{1}\right)^{2}\right| \vec{p}-\vec{p}_{1}\left|Y_{1}^{\mu}\left(\vec{p}-\vec{p}_{1}\right)\right| p_{1} j_{1} m_{1}\right\rangle_{2}
$$




$$
\begin{aligned}
& =\frac{4 \pi}{3}{ }_{2}\left\langle p j m\left|\left(\vec{p}-\vec{p}_{1}\right)^{2} \frac{F^{2}\left(\vec{p}-\vec{p}_{1}\right)^{2}}{\left(\vec{p}-\vec{p}_{1}\right)^{2}+m_{\pi}^{2}}\left\{\sigma(3), \mathcal{Y}_{00}^{0}\left(\overrightarrow{p-\vec{p}_{1}}\right)\right\}^{1, \mu}\right| p_{1} j_{1} m_{1}\right\rangle_{2} \\
& -\frac{4 \pi}{3} 4 \sqrt{15}{ }_{2}\langle p j m| \frac{F^{2}\left(\vec{p}-\vec{p}_{1}\right)^{2}}{\left(\vec{p}-\vec{p}_{1}\right)^{2}+m_{\pi}^{2}} \\
& \times \sum_{a+b=2} \frac{p^{a}\left(-p_{1}\right)^{b}}{\sqrt{\hat{a} ! \hat{b} !}}\left\{\sigma(3), \mathcal{Y}_{a b}^{2}\left(\vec{p}-\vec{p}_{1}\right)\right\}^{1, \mu}\left|p_{1} j_{1} m_{1}\right\rangle_{2}
\end{aligned}
$$

The expansion into Legendre polynomials for the first term is the same as for the c-term and for the second term as for the a-term. We get

$$
\begin{aligned}
M_{b}^{j, 2} & =\frac{8 \pi^{2}}{3} \sum_{\bar{l}}(-)^{\bar{l}} \sqrt{\hat{\bar{l}}} \tilde{H}_{\bar{l}}\left(p, p_{1}\right){ }_{2}\left\langle j m\left|\mathcal{Y}_{\overline{l l}}^{00}\left(\hat{p}, \hat{p}_{1}\right)\left\{\sigma(3), \mathcal{Y}_{00}^{0}\left(\hat{p}, \hat{p}_{1}\right)\right\}^{1, \mu}\right| j_{1} m_{1}\right\rangle_{2} \\
& -\frac{8 \pi^{2}}{3} 4 \sqrt{15} \sum_{\bar{l}}(-)^{\bar{l}} \sqrt{\hat{\bar{l}}} H_{\bar{l}}\left(p, p_{1}\right) \\
& \times \sum_{a+b=2} \frac{p^{a}\left(-p_{1}\right)^{b}}{\sqrt{\hat{a} ! \hat{b} !}}{ }_{2}\left\langle j m\left|\mathcal{Y}_{\overline{l l}}^{00}\left(\hat{p}, \hat{p}_{1}\right)\left\{\sigma(3), \boldsymbol{Y}_{a b}^{2}\left(\hat{p}, \hat{p}_{1}\right)\right\}^{1, \mu}\right| j_{1} m_{1}\right\rangle_{2}
\end{aligned}
$$

To proceed further we have to recouple

$$
\begin{aligned}
& \boldsymbol{Y}_{\overline{l l}}^{00}\left(\hat{p}, \hat{p}_{1}\right)\left\{\sigma(3), \mathcal{Y}_{a b}^{2}\left(\hat{p}, \hat{p}_{1}\right)\right\}^{1, \mu} \\
= & \frac{1}{4 \pi} \sqrt{\hat{a} \hat{b} \hat{b}} \sum_{i_{3} i_{4}}(-)^{i_{4}+a+\bar{l}}\left\{\begin{array}{ccc}
b & a & 2 \\
i_{3} & i_{4} & \bar{l}
\end{array}\right\} \\
\times & C\left(a 0 \bar{l} 0, i_{3} 0\right) C\left(b 0 \bar{l} 0, i_{4} 0\right)\left\{\sigma(3), \mathcal{Y}_{i_{3} i_{4}}^{2}\left(\hat{p}, \hat{p}_{1}\right)\right\}^{1, \mu}
\end{aligned}
$$

and

$$
y_{\frac{00}{l l}}^{0}\left(\hat{p}, \hat{p}_{1}\right)\left\{\sigma(3), \boldsymbol{Y}_{00}^{0}\left(\hat{p}, \hat{p}_{1}\right)\right\}^{1, \mu}=\frac{1}{4 \pi}\left\{\sigma(3), \boldsymbol{Y}_{\overline{l l}}^{0}\left(\hat{p}, \hat{p}_{1}\right)\right\}^{1, \mu}
$$

which leads directly to the following two matrix elements:

$$
\begin{aligned}
& { }_{2}\left\langle j m\left|\left\{\sigma(3), \boldsymbol{Y}_{i_{3} i_{4}}^{2}\left(\hat{p}, \hat{p}_{1}\right)\right\}^{1, \mu}\right| j_{1} m_{1}\right\rangle_{2} \\
= & \delta_{i_{3} l} \delta_{i_{4} l_{1}} C\left(1 \mu j_{1} m_{1}, j m\right) 3 \sqrt{10}(-)^{s-l+1} \sqrt{\hat{j}_{1} \hat{s} \hat{s}_{1}}\left\{\begin{array}{ccc}
\frac{1}{2} & \frac{1}{2} & s_{1} \\
1 & s & \frac{1}{2}
\end{array}\right\}\left\{\begin{array}{ccc}
2 & 1 & 1 \\
l_{1} & s_{1} & j_{1} \\
l & s & j
\end{array}\right\}
\end{aligned}
$$

and

$$
\begin{aligned}
& { }_{2}\left\langle j m\left|\left\{\sigma(3), \boldsymbol{Y}_{\frac{l l}{l l}}^{0}\left(\hat{p}, \hat{p}_{1}\right)\right\}^{1, \mu}\right| j_{1} m_{1}\right\rangle_{2} \\
= & \delta_{\overline{l l} l} \delta_{\bar{l} l_{1}} C\left(1 \mu j_{1} m_{1}, j m\right) \sqrt{6}(-)^{j_{1}} \frac{\sqrt{\hat{j_{1}} \hat{s} \hat{s}_{1}}}{\sqrt{\hat{l}_{1}}}\left\{\begin{array}{ccc}
\frac{1}{2} & \frac{1}{2} & s_{1} \\
1 & s & \frac{1}{2}
\end{array}\right\}\left\{\begin{array}{ccc}
j & j_{1} & 1 \\
s_{1} & s & l
\end{array}\right\}
\end{aligned}
$$


and then to

$$
\begin{aligned}
M_{b}^{j, 2} & =C\left(1 \mu j_{1} m_{1}, j m\right) \\
& \times\left[\delta_{l l_{1}} \frac{2 \pi}{3} \sqrt{6}(-)^{l+j_{1}} \sqrt{\hat{j}_{1} \hat{s} \hat{s}_{1}} \tilde{H}_{l}\left(p, p_{1}\right)\left\{\begin{array}{ccc}
\frac{1}{2} & \frac{1}{2} & s_{1} \\
1 & s & \frac{1}{2}
\end{array}\right\}\left\{\begin{array}{lll}
j & j_{1} & 1 \\
s_{1} & s & l
\end{array}\right\}\right. \\
& -40 \pi \sqrt{6}(-)^{s+1} \sqrt{\hat{j}_{1} \hat{s} \hat{s}_{1}}\left\{\begin{array}{ccc}
\frac{1}{2} & \frac{1}{2} & s_{1} \\
1 & s & \frac{1}{2}
\end{array}\right\}\left\{\begin{array}{ccc}
2 & 1 & 1 \\
l_{1} & s_{1} & j_{1} \\
l & s & j
\end{array}\right\} \\
& \left.\times \sum_{\bar{l}} \hat{\bar{l}} H_{\bar{l}}\left(p, p_{1}\right) \sum_{a+b=2} \frac{p^{a} p_{1}^{b}}{\sqrt{(2 a) !(2 b) !}}\left\{\begin{array}{lll}
b & a & 2 \\
l & l_{1} & \bar{l}
\end{array}\right\} C(a 0 \bar{l} 0, l 0) C\left(b 0 \bar{l} 0, l_{1} 0\right)\right]
\end{aligned}
$$

The summation over the magnetic quantum numbers in eq. (62) using the $m, m_{1}$ dependence from eq. (70) can be done analytically:

$$
\begin{aligned}
& \sum_{m m_{1}} C(j m I M-m, J M) C\left(j_{1} m_{1} I_{1} M_{1}-m_{1}, J_{1} M_{1}\right) C\left(1 \mu j_{1} m_{1}, j m\right) \\
= & (-)^{1+j_{1}+I+J} \sqrt{\hat{j} \hat{J}_{1}} C\left(1 \mu J_{1} M_{1}, J M\right)\left\{\begin{array}{ccc}
1 & j_{1} & j \\
I & J & J_{1}
\end{array}\right\}
\end{aligned}
$$

and we get for $M_{b}^{J, 2}$

$$
\begin{aligned}
M_{b}^{J, 2} & =\frac{\delta\left(q-q_{1}\right)}{q^{2}} \delta_{\lambda \lambda_{1}} \delta_{I I_{1}} C\left(1 \mu J_{1} M_{1}, J M\right) \\
& \times(-)^{I+J} \sqrt{\hat{j} \hat{j}_{1} \hat{s} \hat{s}_{1} \hat{J}_{1}}\left\{\begin{array}{ccc}
\frac{1}{2} & \frac{1}{2} & s_{1} \\
1 & s & \frac{1}{2}
\end{array}\right\}\left\{\begin{array}{ccc}
1 & j_{1} & j \\
I & J & J_{1}
\end{array}\right\} \\
& \times\left[\delta_{l l_{1}} \frac{2 \pi}{3} \sqrt{6}(-)^{l+1} \tilde{H}_{l}\left(p, p_{1}\right)\left\{\begin{array}{ccc}
j & j_{1} & 1 \\
s_{1} & s & l
\end{array}\right\}\right. \\
& -40 \pi \sqrt{6}(-)^{s+j_{1}}\left\{\begin{array}{ccc}
2 & 1 & 1 \\
l_{1} & s_{1} & j_{1} \\
l & s & j
\end{array}\right\} \\
& \left.\times \sum_{\bar{l}} \hat{\bar{l}} H_{\bar{l}}\left(p, p_{1}\right) \sum_{a+b=2} \frac{p^{a} p_{1}^{b}}{\sqrt{(2 a) !(2 b) !}}\left\{\begin{array}{lll}
b & a & 2 \\
l & l_{1} & \bar{l}
\end{array}\right\} C(a 0 \bar{l} 0, l 0) C\left(b 0 \bar{l} 0, l_{1} 0\right)\right]
\end{aligned}
$$

The evaluation of $M_{b}^{J, 3}$ proceeds along similar lines or can be abbreviated by judicious replacements and using symmetries of $6 \mathrm{j}$ - and $9 \mathrm{j}$-symbols. The result is

$$
M_{b}^{J, 3}=\frac{\delta\left(q^{\prime}-q_{2}\right)}{q^{2}} \delta_{\lambda^{\prime} \lambda_{2}} \delta_{I^{\prime} I_{2}} C\left(1-\mu J^{\prime} M^{\prime}, J_{2} M_{2}\right)
$$




$$
\begin{aligned}
& \times(-)^{I^{\prime}+J_{2}+s^{\prime}-s_{2}} \sqrt{\hat{j}^{\prime} \hat{j}_{2} \hat{s}^{\prime} \hat{s}_{2} \hat{J}^{\prime}}\left\{\begin{array}{ccc}
\frac{1}{2} & \frac{1}{2} & s_{2} \\
1 & s^{\prime} & \frac{1}{2}
\end{array}\right\}\left\{\begin{array}{lll}
1 & j_{2} & j^{\prime} \\
I^{\prime} & J^{\prime} & J_{2}
\end{array}\right\} \\
& \times\left[\delta_{l^{\prime} l_{2}} \frac{2 \pi}{3} \sqrt{6}(-)^{l^{\prime}+1} \tilde{H}_{l^{\prime}}\left(p^{\prime} p_{2}\right)\left\{\begin{array}{ccc}
j^{\prime} & j_{2} & 1 \\
s_{2} & s^{\prime} & l^{\prime}
\end{array}\right\}\right. \\
& -40 \pi \sqrt{6}(-)^{s^{\prime}+j_{2}}\left\{\begin{array}{ccc}
2 & 1 & 1 \\
l_{2} & s_{2} & j_{2} \\
l^{\prime} & s^{\prime} & j^{\prime}
\end{array}\right\} \\
& \left.\times \sum_{\bar{l}} \hat{\bar{l}} H_{\bar{l}}\left(p^{\prime} p_{2}\right) \sum_{a+b=2} \frac{p^{\prime a} p_{2}^{b}}{\sqrt{(2 a) !(2 b) !}}\left\{\begin{array}{lll}
b & a & 2 \\
l^{\prime} & l_{2} & \bar{l}
\end{array}\right\} C\left(a 0 \bar{l} 0, l^{\prime} 0\right) C\left(b 0 \bar{l} 0, l_{2} 0\right)\right]
\end{aligned}
$$

Now, looking back to eq. (60), we can perform the sum over $\mu$ :

$$
\sum_{\mu}(-)^{\mu} C\left(1 \mu J_{1} m_{1}, J M\right) C\left(1-\mu J^{\prime} M^{\prime}, J_{1} M_{1}\right)=\delta_{J J^{\prime}} \delta_{M M^{\prime}}(-)^{J_{1}-J} \sqrt{\frac{\hat{J}_{1}}{\hat{J}}}
$$

Thereby we used $J_{1}=J_{2}$ and $M_{1}=M_{2}$, which results from the fact that the permutation operator standing between $M_{b}^{J, 2}$ and $M_{b}^{J, 3}$ (see eq. (35)) conserves $J$ and $M$. Thus despite the fact that $M_{b}^{J, 2}$ and $M_{b}^{J, 3}$ do not conserve $J$, as is obvious from the Clebsch-Gordan coefficients in eq. (74), the total expression $\left.v_{4}^{(1)}\right|_{b}$ does it, as expected. The parity, however, is conserved by the individual quantities $M_{b}^{J, 2}$ and $M_{b}^{J, 3}$.

Having performed the $\mu$-summation reduced $M$-quantities occur, which are defined as

$$
\begin{aligned}
& M_{b}^{J, 2} \equiv C\left(1 \mu J_{1} M_{1}, J M\right)(-)^{J_{1}-J} \bar{M}_{b}^{J, 2} \\
& M_{b}^{J, 3} \equiv C\left(1-\mu J^{\prime} M^{\prime}, J_{2} M_{2}\right) \sqrt{\frac{\hat{J}^{\prime}}{\hat{J}_{2}}} \bar{M}_{b}^{J, 3}
\end{aligned}
$$

and which obey the following symmetry relation

$$
\bar{M}_{b}^{J, 2}\left(p \alpha, p_{1} \alpha_{1}\right)=(-)^{s_{1}-s} \bar{M}_{b}^{J, 3}\left(p_{1} \alpha_{1}, p \alpha\right)
$$

Again we see that the expressions using this new PWD, now for the $b$-term, are much simpler than the expressions of the old PWD, see [9] and [15].

The last term, the $d$-term, contains the operator $\vec{\sigma}_{1} \cdot \vec{Q} \times{\overrightarrow{Q^{\prime}}}^{\prime}$ (see eq. (25)):

$$
\vec{\sigma}_{1} \cdot \vec{Q} \times \vec{Q}^{\prime}=\vec{\sigma}_{1} \cdot(i \sqrt{2})\left\{Q_{1}, Q_{1}^{\prime}\right\}^{1}=i \sqrt{6} \frac{4 \pi}{3} Q Q^{\prime}\left\{\sigma(1),\left\{Y_{1}(\hat{Q}), Y_{1}\left(\hat{Q}^{\prime}\right)\right\}^{1}\right\}^{0,0}
$$




$$
\begin{aligned}
& =-i \sqrt{2} \frac{4 \pi}{3} Q Q^{\prime} \sum_{\mu}(-)^{\mu}\left\{\sigma(1), Y_{1}(\hat{Q})\right\}^{1,-\mu} Y_{1}^{\mu}\left(\hat{Q}^{\prime}\right) \\
& =i \sqrt{2} \frac{4 \pi}{3} Q Q^{\prime} \sum_{\mu}(-)^{\mu}\left\{\sigma(1), Y_{1}\left(\hat{Q}^{\prime}\right)\right\}^{1,-\mu} Y_{1}^{\mu}(\hat{Q})
\end{aligned}
$$

According to the two possibilities to couple $\sigma(1)$ we have two ways to write down the $d$-term:

$$
\begin{aligned}
{ }_{1}\left\langle p q \alpha\left|v_{4}^{(1)}\right|^{d} \mid p^{\prime} q^{\prime} \alpha^{\prime}\right\rangle_{3} & =P_{1 \leftrightarrow 2} \sum_{\mu}(-)^{\mu} M_{d}^{J, 2} P_{2 \leftrightarrow 3} M_{b}^{J, 3} \\
& =P_{1 \leftrightarrow 2} \sum_{\mu}(-)^{\mu} M_{b}^{J, 2} P_{2 \leftrightarrow 3} M_{d}^{J, 3}
\end{aligned}
$$

with

$$
\begin{aligned}
& M_{d}^{J, 2} \equiv-i \sqrt{2} \sqrt{\frac{4 \pi}{3}}{ }_{2}\left\langle p q \alpha_{J}\left|Q\left\{\sigma(1), Y_{1}(\hat{Q})\right\}^{1 \mu} F^{2}\left(\vec{Q}^{2}\right) \frac{\vec{\sigma}_{3} \cdot \vec{Q}}{\vec{Q}^{2}+m_{\pi}^{2}}\right| p_{1} q_{1} \alpha_{1 J}\right\rangle_{2} \\
& M_{d}^{J, 3} \equiv i \sqrt{2} \sqrt{\frac{4 \pi}{3}}{ }_{3}\left\langle p_{2} q_{2} \alpha_{2 J}\left|Q^{\prime}\left\{\sigma(1), Y_{1}\left(\hat{Q}^{\prime}\right)\right\}^{1,-\mu} F^{2}\left({\overrightarrow{Q^{\prime}}}^{2}\right) \frac{\vec{\sigma}_{2} \cdot \vec{Q}^{\prime}}{{\overrightarrow{Q^{\prime}}}^{2}+m_{\pi}^{2}}\right| p^{\prime} q^{\prime} \alpha_{J}^{\prime}\right\rangle_{3}
\end{aligned}
$$

The permutation operators $P_{1 \leftrightarrow 2}$ and $P_{2 \leftrightarrow 3}$ in eq. (78) stand for the recoupling matrix elements (see eq. (35)) and we dropped the iso-spin factor $I_{4}^{(1)}$. We see that $M_{d}^{J, 2}$ and $M_{d}^{J, 3}$ are purely imaginary. Together with the imaginary iso-spin factor of eq. (34), the matrix element is of course real.

For $M_{d}^{J, 2}$ we have to recouple the operator

$$
\begin{aligned}
& \vec{\sigma}_{3} \cdot \vec{Q}\left\{\sigma(1), Y_{1}(\hat{Q})\right\}^{1 \mu}=\vec{\sigma}_{3} \cdot\left(\vec{p}-\vec{p}_{1}\right)\left\{\sigma(1), Y_{1}\left(\overrightarrow{p-\vec{p}_{1}}\right)\right\}^{1 \mu} \\
= & -\sqrt{3} \sqrt{\frac{4 \pi}{3}}\left|\vec{p}-\vec{p}_{1}\right|\left\{\left\{\sigma(3), Y_{1}\left(\overrightarrow{p-\vec{p}_{1}}\right)\right\}^{0},\left\{\sigma(1), Y_{1}\left(\overrightarrow{p-\vec{p}_{1}}\right)\right\}^{1}\right\}^{1 \mu} \\
= & \sqrt{3}\left|\vec{p}-\vec{p}_{1}\right| \sum_{i_{1} i_{2}}(-)^{i_{2}} \sqrt{\hat{i}_{1}} C\left(1010, i_{2} 0\right)\left\{\begin{array}{ccc}
i_{2} & i_{1} & 1 \\
1 & 1 & 1
\end{array}\right\} \\
\times & \left\{\{\sigma(1), \sigma(3)\}^{i_{1}}, Y_{i_{2}}\left(\vec{p}-\vec{p}_{1}\right)\right\}^{1 \mu} \\
= & \frac{1}{\sqrt{3}}\left|\vec{p}-\vec{p}_{1}\right|\left\{\{\sigma(1), \sigma(3)\}^{1}, Y_{0}\left(\overrightarrow{p-\vec{p}_{1}}\right)\right\}^{1 \mu} \\
+ & \sqrt{2}\left|\vec{p}-\vec{p}_{1}\right| \sum_{i_{1}=1,2} \sqrt{\hat{i}_{1}}\left\{\begin{array}{ccc}
2 & i_{1} & 1 \\
1 & 1 & 1
\end{array}\right\}\left\{\{\sigma(1), \sigma(3)\}^{i_{1}}, Y_{2}\left(\vec{p}-\vec{p}_{1}\right)\right\}^{1 \mu}
\end{aligned}
$$

In the last step we inserted explicitly the sum over the two $i_{2}$-values. Finally we decompose the spherical harmonics and get 


$$
\begin{aligned}
& \vec{\sigma}_{3} \cdot \vec{Q}\left\{\sigma(1), Y_{1}(\hat{Q})\right\}^{1 \mu} \\
= & \sqrt{\frac{4 \pi}{3}}\left|\vec{p}-\vec{p}_{1}\right|\left\{\{\sigma(1), \sigma(3)\}^{1}, y_{00}^{0}\left(\hat{p}, \hat{p}_{1}\right)\right\}^{1 \mu} \\
+ & 8 \sqrt{15 \pi} \frac{1}{\left|\vec{p}-\vec{p}_{1}\right|} \sum_{i_{1}=1,2} \sqrt{\hat{i}_{1}}\left\{\begin{array}{ccc}
2 & i_{1} & 1 \\
1 & 1 & 1
\end{array}\right\} \\
\times & \sum_{a+b=2} \frac{p^{a}\left(-p_{1}\right)^{b}}{\sqrt{\hat{a} ! \hat{b} !}}\left\{\{\sigma(1), \sigma(3)\}^{i_{1}}, y_{a b}^{2}\left(\hat{p}, \hat{p}_{1}\right)\right\}^{1 \mu}
\end{aligned}
$$

which inserted into $M_{d}^{J, 2}$ yields:

$$
\begin{aligned}
M_{d}^{J, 2} & =-i \sqrt{2} \frac{4 \pi}{3}{ }_{2}\left\langle p q \alpha_{J}\left|\frac{F^{2}\left(\left|\vec{p}-\vec{p}_{1}\right|^{2}\right)}{\left|\vec{p}-\vec{p}_{1}\right|^{2}+m_{\pi}^{2}}\right| \vec{p}-\left.\vec{p}_{1}\right|^{2}\left\{\{\sigma(1), \sigma(3)\}^{1}, y_{00}^{0}\left(\hat{p}, \hat{p}_{1}\right)\right\}^{1 \mu} \mid p_{1} q_{1} \alpha_{1 J}\right\rangle_{2} \\
& -i 16 \pi \sqrt{10} \sum_{i_{1}=1,2} \sqrt{\hat{i}_{1}}\left\{\begin{array}{ccc}
2 & i_{1} & 1 \\
1 & 1 & 1
\end{array}\right\} \sum_{a+b=2} \frac{p^{a}\left(-p_{1}\right)^{b}}{\sqrt{\hat{a} ! \hat{b} !}} \\
& \times{ }_{2}\left\langle p q \alpha_{J}\left|\frac{F^{2}\left(\left|\vec{p}-\vec{p}_{1}\right|^{2}\right)}{\left|\vec{p}-\vec{p}_{1}\right|^{2}+m_{\pi}^{2}}\left\{\{\sigma(1), \sigma(3)\}^{i_{1}}, y_{a b}^{2}\left(\hat{p}, \hat{p}_{1}\right)\right\}^{1 \mu}\right| p_{1} q_{1} \alpha_{1 J}\right\rangle_{2}
\end{aligned}
$$

This expression contains no singularity.

It remains to expand the $x$-dependence in terms of Legendre polynomials, which leads to

$$
\begin{aligned}
M_{d}^{J, 2} & =-i \sqrt{2} \frac{8 \pi^{2}}{3} \sum_{\bar{l}} \sqrt{\hat{\bar{l}}}(-)^{\bar{l}} \tilde{H}_{\bar{l}}\left(p, p_{1}\right) \\
& \times{ }_{2}\left\langle p q \alpha_{J}\left|\mathcal{Y}_{\overline{l l}}^{0}\left(\hat{p}, \hat{p}_{1}\right)\left\{\{\sigma(1), \sigma(3)\}^{1}, \mathcal{Y}_{00}^{0}\left(\hat{p}, \hat{p}_{1}\right)\right\}^{1 \mu}\right| p_{1} q_{1} \alpha_{1 J}\right\rangle_{2} \\
& -i 32 \pi^{2} \sqrt{10} \sum_{i_{1}=1,2} \sqrt{\hat{i}_{1}}\left\{\begin{array}{ccc}
2 & i_{1} & 1 \\
1 & 1 & 1
\end{array}\right\} \sum_{a+b=2} \frac{p^{a}\left(-p_{1}\right)^{b}}{\sqrt{\hat{a} ! \hat{b} !}} \sum_{\bar{l}} \sqrt{\hat{\bar{l}}}(-)^{\bar{l}} H_{\bar{l}}\left(p, p_{1}\right) \\
& \times{ }_{2}\left\langle p q \alpha_{J}\left|\mathcal{Y}_{\frac{0}{l l}}^{0}\left(\hat{p}, \hat{p}_{1}\right)\left\{\{\sigma(1), \sigma(3)\}^{i_{1}}, \mathcal{Y}_{a b}^{2}\left(\hat{p}, \hat{p}_{1}\right)\right\}^{1 \mu}\right| p_{1} q_{1} \alpha_{1 J}\right\rangle_{2}
\end{aligned}
$$

and to combine the spherical harmonics

$$
\begin{aligned}
& \mathcal{Y}_{\overline{l l}}^{00}\left(\hat{p}, \hat{p}_{1}\right)\left\{\{\sigma(1), \sigma(3)\}^{i_{1}}, \mathcal{Y}_{a b}^{2}\left(\hat{p}, \hat{p}_{1}\right)\right\}^{1 \mu} \\
= & \left\{\left\{\{\sigma(1), \sigma(3)\}^{i_{1}}, \mathcal{Y}_{a b}^{2}\left(\hat{p}, \hat{p}_{1}\right)\right\}^{1} \mathcal{Y}_{\overline{l l}}^{0}\left(\hat{p}, \hat{p}_{1}\right)\right\}^{1 \mu} \\
= & \sum_{i_{3} i_{4}} \frac{1}{4 \pi}(-)^{\bar{l}+i_{4}+a} \sqrt{\hat{a} \hat{b} \hat{\bar{l}}} C\left(a 0 \bar{l} 0, i_{3} 0\right) C\left(b 0 \bar{l} 0, i_{4} 0\right) \\
\times & \left\{\begin{array}{ccc}
a & b & 2 \\
i_{4} & i_{3} & \bar{l}
\end{array}\right\}\left\{\{\sigma(1), \sigma(3)\}^{i_{1}}, \mathcal{Y}_{i_{3} i_{4}}^{2}\left(\hat{p}, \hat{p}_{1}\right)\right\}^{1 \mu}
\end{aligned}
$$


and

$$
\mathcal{Y}_{\frac{00}{l l}}^{0}\left(\hat{p}, \hat{p}_{1}\right)\left\{\{\sigma(1), \sigma(3)\}^{1}, \boldsymbol{Y}_{00}^{0}\left(\hat{p}, \hat{p}_{1}\right)\right\}^{1 \mu}=\frac{1}{4 \pi}\left\{\{\sigma(1), \sigma(3)\}^{1}, \mathcal{Y}_{\frac{0}{l l}}^{0}\left(\hat{p}, \hat{p}_{1}\right)\right\}^{1 \mu}
$$

Thus we have to evaluate two matrix elements. The first is

$$
\begin{aligned}
& { }_{2}\left\langle p q \alpha\left|\left\{\{\sigma(1), \sigma(3)\}^{i_{1}}, \mathcal{Y}_{i_{3} i_{4}}^{2}\left(\hat{p}, \hat{p}_{1}\right)\right\}^{1 \mu}\right| p_{1} q_{1} \alpha_{1}\right\rangle_{2} \\
= & \frac{\delta\left(q-q_{1}\right)}{q^{2}} \delta_{\lambda \lambda_{1}} \delta_{I I_{1}} \sum_{m m_{1}} C(j m I M-m, J M) C\left(j_{1} m_{1} I_{1} M_{1}-m_{1}, J_{1} M_{1}\right) \\
\times & C\left(1 \mu j_{1} m_{1}, j m\right)(-)^{i_{1}+1} \sqrt{3 \hat{j_{1}} \hat{l} \hat{s}}\left\{\begin{array}{ccc}
2 & i_{1} & 1 \\
l_{1} & s_{1} & j_{1} \\
l & s & j
\end{array}\right\} \\
\times & { }_{2}\left\langle l\left\|\mathcal{Y}_{i_{3} i_{4}}^{2}\left(\hat{p}, \hat{p}_{1}\right)\right\| l_{1}\right\rangle_{2}{ }_{2}\left\langle s\left\|\{\sigma(1), \sigma(3)\}^{i_{1}}\right\| s_{1}\right\rangle_{2}
\end{aligned}
$$

Only the reduced spin matrix element is new:

$$
{ }_{2}\left\langle s\left\|\{\sigma(1), \sigma(3)\}^{i_{1}}\right\| s_{1}\right\rangle_{2}=6 \sqrt{\hat{i}_{1} \hat{s}_{1}}\left\{\begin{array}{ccc}
1 & 1 & i_{1} \\
\frac{1}{2} & \frac{1}{2} & s_{1} \\
\frac{1}{2} & \frac{1}{2} & s
\end{array}\right\}
$$

After summation over $m$ and $m_{1}$ we get

$$
\begin{aligned}
& { }_{2}\left\langle p q \alpha\left|\left\{\{\sigma(1), \sigma(3)\}^{i_{1}}, \mathcal{Y}_{i_{3} i_{4}}^{2}\left(\hat{p}, \hat{p}_{1}\right)\right\}^{1 \mu}\right| p_{1} q_{1} \alpha_{1}\right\rangle_{2} \\
& =\frac{\delta\left(q-q_{1}\right)}{q^{2}} \delta_{\lambda \lambda_{1}} \delta_{I I_{1}} \delta_{i_{3} l} \delta_{i_{4} l_{1}} 6 \sqrt{15}(-)^{j_{1}-l+I+J+i_{1}} \sqrt{\hat{j} \hat{j}_{1} \hat{s} \hat{s}_{1} \hat{J}_{1} \hat{i}_{1}} \\
& \times C\left(1 \mu J_{1} M_{1}, J M\right)\left\{\begin{array}{ccc}
1 & j_{1} & j \\
I & J & J_{1}
\end{array}\right\}\left\{\begin{array}{ccc}
2 & i_{1} & 1 \\
l_{1} & s_{1} & j_{1} \\
l & s & j
\end{array}\right\}\left\{\begin{array}{ccc}
1 & 1 & i_{1} \\
\frac{1}{2} & \frac{1}{2} & s_{1} \\
\frac{1}{2} & \frac{1}{2} & s
\end{array}\right\}
\end{aligned}
$$

The second matrix element is

$$
\begin{aligned}
& { }_{2}\left\langle p q \alpha\left|\left\{\{\sigma(1), \sigma(3)\}^{1}, Y_{\frac{l l}{l l}}^{0}\left(\hat{p}, \hat{p}_{1}\right)\right\}^{1 \mu}\right| p_{1} q_{1} \alpha_{1}\right\rangle_{2} \\
= & \frac{\delta\left(q-q_{1}\right)}{q^{2}} \delta_{\lambda \lambda_{1}} \delta_{I I_{1}} \delta_{\overline{l l}} \delta_{\overline{l l}_{1}} 6 \sqrt{3}(-)^{s+I+J} \frac{\sqrt{\hat{j} \hat{j}_{1} \hat{s} \hat{s}_{1} \hat{J}_{1}}}{\sqrt{\hat{l}}} \\
\times & C\left(1 \mu J_{1} M_{1}, J M\right)\left\{\begin{array}{ccc}
1 & j_{1} & j \\
I & J & J_{1}
\end{array}\right\}\left\{\begin{array}{ccc}
l & s & j \\
1 & j_{1} & s_{1}
\end{array}\right\}\left\{\begin{array}{ccc}
1 & 1 & 1 \\
\frac{1}{2} & \frac{1}{2} & s_{1} \\
\frac{1}{2} & \frac{1}{2} & s
\end{array}\right\}
\end{aligned}
$$


We end up with

$$
\begin{aligned}
& M_{d}^{J, 2}=\frac{\delta\left(q-q_{1}\right)}{q^{2}} \delta_{\lambda \lambda_{1}} \delta_{I I_{1}}(-)^{I+J+1} \sqrt{\hat{j} \hat{j}_{1} \hat{s} \hat{s}_{1} \hat{J}_{1}} C\left(1 \mu J_{1} M_{1}, J M\right)\left\{\begin{array}{ccc}
1 & j_{1} & j \\
I & J & J_{1}
\end{array}\right\}
\end{aligned}
$$

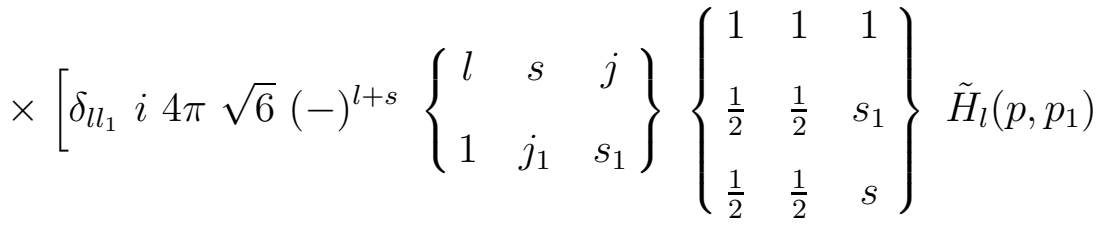

$$
\begin{aligned}
& +i 240 \pi \sqrt{6}(-)^{j_{1}} \sum_{i_{1}}(-)^{i_{1}} \hat{i}_{1}\left\{\begin{array}{ccc}
2 & i_{1} & 1 \\
1 & 1 & 1
\end{array}\right\}\left\{\begin{array}{ccc}
2 & i_{1} & 1 \\
l_{1} & s_{1} & j_{1} \\
l & s & j
\end{array}\right\}\left\{\begin{array}{ccc}
1 & 1 & i_{1} \\
\frac{1}{2} & \frac{1}{2} & s_{1} \\
\frac{1}{2} & \frac{1}{2} & s
\end{array}\right\} \\
& \left.\times \sum_{a+b=2} \frac{p^{a} p_{1}^{b}}{\sqrt{(2 a) !(2 b) !}} \sum_{\bar{l}} \hat{\bar{l}} H_{\bar{l}}\left(p, p_{1}\right)\left\{\begin{array}{lll}
a & b & 2 \\
l_{1} & l & \bar{l}
\end{array}\right\} C(a 0 \bar{l} 0, l 0) C\left(b 0 \bar{l} 0, l_{1} 0\right)\right]
\end{aligned}
$$

Similar steps or using judicious replacements lead to

$$
\begin{aligned}
& M_{d}^{J, 3}=\frac{\delta\left(q^{\prime}-q_{2}\right)}{q^{\prime 2}} \delta_{\lambda^{\prime} \lambda_{2}} \delta_{I^{\prime} I_{2}} \\
& \times(-)^{I^{\prime}+J_{2}+l^{\prime}+l_{2}+1} \sqrt{\hat{j}^{\prime} \hat{j}_{2} \hat{s}^{\prime} \hat{s}_{2} \hat{J}^{\prime}} C\left(1-\mu J^{\prime} M^{\prime}, J_{2} M_{2}\right)\left\{\begin{array}{ccc}
1 & j_{2} & j^{\prime} \\
I^{\prime} & J^{\prime} & J_{2}
\end{array}\right\} \\
& \times\left[\delta_{l^{\prime} l_{2}} i 4 \pi \sqrt{6}(-)^{l^{\prime}+s^{\prime}}\left\{\begin{array}{ccc}
l^{\prime} & s^{\prime} & j^{\prime} \\
1 & j_{2} & s_{2}
\end{array}\right\}\left\{\begin{array}{ccc}
1 & 1 & 1 \\
\frac{1}{2} & \frac{1}{2} & s_{2} \\
\frac{1}{2} & \frac{1}{2} & s^{\prime}
\end{array}\right\} \tilde{H}_{l^{\prime}}\left(p^{\prime} p_{2}\right)\right. \\
& +i 240 \pi \sqrt{6}(-)^{j_{2}} \sum_{i_{1}}(-)^{i_{1}} \hat{i}_{1}\left\{\begin{array}{ccc}
2 & i_{1} & 1 \\
1 & 1 & 1
\end{array}\right\}\left\{\begin{array}{ccc}
2 & i_{1} & 1 \\
l_{2} & s_{2} & j_{2} \\
l^{\prime} & s^{\prime} & j^{\prime}
\end{array}\right\}\left\{\begin{array}{ccc}
1 & 1 & i_{1} \\
\frac{1}{2} & \frac{1}{2} & s_{2} \\
\frac{1}{2} & \frac{1}{2} & s^{\prime}
\end{array}\right\} \\
& \left.\times \sum_{a+b=2} \frac{p^{a} p_{2}^{b}}{\sqrt{(2 a) !(2 b) !}} \sum_{\bar{l}} \hat{\bar{l}} H_{\bar{l}}\left(p^{\prime} p_{2}\right)\left\{\begin{array}{ccc}
a & b & 2 \\
l_{2} & l^{\prime} & \bar{l}
\end{array}\right\} C\left(a 0 \bar{l} 0, l^{\prime} 0\right) C\left(b 0 \bar{l} 0, l_{2} 0\right)\right]
\end{aligned}
$$

The last step is to perform the sum over $\mu$, eq. (78). Because in eq. (78) $M_{d}$ is used together with $M_{b}$, we have to define $\bar{M}_{d}$ consistently with $\bar{M}_{b}$ (eq. (75)):

$$
\begin{aligned}
& M_{d}^{J, 2} \equiv C\left(1 \mu J_{1} M_{1}, J M\right)(-)^{J_{1}-J} \bar{M}_{d}^{J, 2} \\
& M_{d}^{J, 3} \equiv C\left(1-\mu J^{\prime} M^{\prime}, J_{2} M_{2}\right) \sqrt{\frac{\hat{J}^{\prime}}{\hat{J}_{2}}} \bar{M}_{d}^{J, 3}
\end{aligned}
$$

and we have the relation 


$$
\bar{M}_{d}^{J, 2}\left(p \alpha, p_{1} \alpha_{1}\right)=(-)^{l+l_{1}} \bar{M}_{d}^{J, 3}\left(p_{1} \alpha_{1}, p \alpha\right)
$$

Then the summation over $\mu$ can be done analytically using again eq. (74).

This concludes the presentation of the new PWD for all four terms of the TM 3NF. In this new approach one avoids the pitfall of the old PWD and one can evaluate now the 3NF for higher partial waves.

\section{NUMERICAL EXAMPLES}

This section has two aims. The first is to show that the old and new PWD give the same results for partial waves where the old PWD is still numerically valid. The second is to show that the extension of the new PWD to higher partial waves gives correct results. Lower and higher partial waves refer to the outer states of type 1 in the matrix elements of the $3 \mathrm{NF}$. The number of intermediate states of type 2 or 3 in eqs.(31) and (35) are principally unlimited and the necessary number of states for an accurate representation of the $3 \mathrm{NF}$ matrix elements have to be determined numerically. We find, that in eqs.(31) and (35) the $\alpha^{\prime \prime}$ sums require two-body angular momenta up to $j_{\max }=5$. As already mentioned the additional sums in eq. (35) over $\alpha_{1 J}$ and $\alpha_{2 J}$ are essentially trivial, since the corresponding

quantum numbers are only marginally changed by the action of $\left.w_{4}^{(1)}\right|_{2,3}$ from the ones of the states of type 2 and 3.

We calculate the $3 \mathrm{NF}$ at $16 p$-points below $10 \mathrm{fm}^{-1}$ and $16 q$-points below $20 \mathrm{fm}^{-1}$. According to our experience this is enough to describe the 3NF [12] [15]. The inner basis states of type 2 and 3 require $20 p$ - and $q$-points in order to achieve a fully converged result. The $p$-range is extended to $16 \mathrm{fm}^{-1}$ in order to avoid extrapolation.

As mentioned in section [1] the old PWD fails for $l \geq 4$ and $\lambda \geq 4$. Therefore the largest two-body angular momentum, for which the old PWD works, is $j=2$. Under this limitation we can compare the old and new PWD. We choose $\Lambda=5.8 m_{\pi}$ in eq. (26) and take as NN force the CD Bonn (np) [18] force restricted to $j \leq 2$, too. 
In Fig. 3 we show two polarization observables, which show a big effect of the $3 \mathrm{NF}$. (The differential cross section is only marginally affected by the 3NF.) Fig. 3 demonstrates that the predictions for the old and new PWD do completely overlap. This is true for all other elastic observables, too.

For the breakup process we calculated cross sections and all analyzing powers, spin correlation coefficients and vector spin transfer coefficients for standard kinematics. Again we chose as examples in Fig. 4 observables with big $3 \mathrm{NF}$ effects. They are both observables under np QFS conditions. Again the agreement of the two curves for the old and new PWD is very good. This is generally true. If one chooses $j_{\max }=4$ for the inner basis states 2 and 3 instead of $j_{\max }=5$ the deviations in the observables to the full result are up to a few percent.

In order to show that also the extension of the new PWD to higher partial waves works well we performed a calculation of the triton binding energy with $j_{\max }=4$. As NN force we used here the AV14 potential [19]. Our result for the triton binding energy is $-9.36 \mathrm{MeV}$, which agrees exactly with the result of the Los Alamos - Iowa group [11].

We obtained the results presented up to now not by using eq. (31) but the form

$$
\begin{aligned}
& { }_{1}\left\langle p q \alpha\left|V_{4}^{(1)}(1+P)\right| p^{\prime} q^{\prime} \alpha^{\prime}\right\rangle_{1} \\
= & \mathcal{Y}^{{ }^{\prime \prime}}{ }_{1}\left\langle p q \alpha\left|V_{4}^{(1)}\right| p^{\prime \prime} q^{\prime \prime} \alpha^{\prime \prime}\right\rangle_{1}{ }_{1}\left\langle p^{\prime \prime} q^{\prime \prime} \alpha^{\prime \prime}|(1+P)| p^{\prime} q^{\prime} \alpha^{\prime}\right\rangle_{1}
\end{aligned}
$$

This is identical to eq. (31) if the $\alpha^{\prime \prime}$ summation is unlimited. In the calculations, however, we restricted the double primed channels to the physical channels, which is an approximation. The old code for the $3 \mathrm{NF}$ did anyhow not allow the higher partial waves. Now using eq. (31) and the new PWD we can check the quality of that approximation. We compare the results for triton binding energies based on the new PWD of the 3NF achieved via eq. (31) (denoted by $E_{b}^{(1)}$ ) with the fully converged $\alpha^{\prime \prime}$-sum to the result gained through eq. (94) (denoted by $\left.E_{b}^{(2)}\right)$, where the $\alpha^{\prime \prime}$-sum is restricted to the same number of partial waves as used in the outer states. The latter number is fixed by choosing a certain maximal total two-body angular momentum $j_{\max }$. Our results are shown in table [. For $j_{\max }=5$ we achieved convergence, 
since the two binding energies $E_{b}^{(1)}$ and $E_{b}^{(2)}$ are equal. The effect of the truncation is large

for $j_{\max }=2$, whereas $E_{b}^{(1)}$ under the same restriction of outer partial waves is already close to the final result. We also see, that the $j_{\max }=4$ result for $E_{b}^{(2)}$ is close to the correct number $E_{b}^{(1)}$.

The results with the $\mathrm{NN}$ force only are nearly converged at $j_{\max }=3$, whereas the interplay with the $3 \mathrm{NF}$ causes a decrease in binding energy for $j_{\max }=3$ and only for $j_{\max }=4$ the final result is essentially reached.

\section{SUMMARY AND OUTLOOK}

We demonstrated in section I1 that the way used up to now to decompose 3NF's in momentum space into partial waves [9] leads unavoidably to numerical instabilities. They occur necessarily if the momenta and the angular momentum quantum numbers are high. The applications carried through up to now are not affected by that numerical instability, since the $3 \mathrm{NF}$ has been applied up to now only in low partial waves. For higher energies, however, high angular momenta are activated and the old PWD cannot be used.

In section III we presented a new PWD. The basic idea is to treat the $3 \mathrm{NF}$ in the example of the $2 \pi$-exchange as if it would be a sequence of two pion exchanges among different pairs. This requires the choice of different Jacobi variables adopted to the two different twobody subsystems. Consequently in between there has to occur a recoupling of the Jacobi variables. In this manner we not only achieve a much simpler analytical form of the partial wave decomposed $3 \mathrm{NF}$, but also an expression, which is free of any artificial singularity and numerically stable in all angular momentum states. The recoupling between the two meson exchanges and also to the external basis states, which require the third possibility for the choice of a two-body subsystem, are performed with the aid of certain permutation operators, which are laid out in appendix A. The application of these permutation operators lead to two-dimensional interpolations, which require very efficient and accurate tools. To that purpose we have rewritten standard hermitian cubic splines into a new form, which is 
shown in appendix B.

Finally we have shown in section $[\nabla]$ two numerical examples for the reliability of the new PWD of the 3NF. We used the TM 3NF model and calculated in the first example observables in elastic nd scattering and the breakup process at $E_{l a b}=10.3 \mathrm{MeV}$. The forces were restricted to $j_{\max }=2$ so that the old PWD could also be used. For all observables we found a very good agreement between the predictions achieved with the old and new PWD. In the second example we extended the new PWD of the TM 3NF to higher partial waves with $j_{\max }=5$ and calculated the triton binding energy. We could confirm the result of the Los Alamos - Iowa group truncated at $j_{\max }=4$. The convergence of the triton binding energies as a function of $j_{\max }$ as displayed in table 1 shows, that in contrast to the case of $\mathrm{NN}$ forces only, the interplay with the $3 \mathrm{NF}$ can lead to a more oscillating approach of the limiting value. More benchmark calculations are underway.

Summarizing we can say that we are now able to calculate the $3 \mathrm{NF}$ up to any partial wave, limited only by computer resources. This new scheme can of course be taken over from the $2 \pi$-exchange, exemplified here, to any other two-meson exchange.

It is interesting to note that the new PWD has still another application. If one wants to calculate three-body meson exchange currents as they occur for example in electron scattering on ${ }^{3} \mathrm{He}\left({ }^{3} \mathrm{H}\right)$ or heavier nuclei one faces exactly the same problems as described here. In order to get correct results for all partial waves one has to follow for the PWD of the three-body meson exchange currents the scheme which we have introduced above.

\section{Acknowledgements}

This work was supported by the Deutsche Forschungsgemeinschaft, the Polish Committee for Scientific Research under Grant No. 2 P302 10406 and the Polish-American Maria Skłodowska-Curie II FUND under Grant No. MEN/NSF-94-161. The numerical calculations have been performed on the CRAY T90 and the CRAY T3E of the Höchstleistungsrechenzentrum in Jülich, Germany. 


\section{APPENDIX A: THE PERMUTATION OPERATORS}

In the Faddeev scheme [12] [13] we apply the permutation operator $P=P_{12} P_{23}+P_{13} P_{23}$ always to states which are anti-symmetric in the two-body subcluster 2-3. Regarding eqs. (35) and (94) we see the recoupling matrix elements ${ }_{1}\left\langle p q \alpha \mid p^{\prime} q^{\prime} \alpha^{\prime}\right\rangle_{2},{ }_{2}\left\langle p q \alpha_{J} \mid p^{\prime} q^{\prime} \alpha_{J}^{\prime}\right\rangle_{3}$, and ${ }_{3}\left\langle p q \alpha \mid p^{\prime} q^{\prime} \alpha^{\prime}\right\rangle_{1}$. Since |\rangle$_{3} \equiv P_{13} P_{23}|\rangle_{1}$ and |\rangle$_{2} \equiv P_{12} P_{23}|\rangle_{1}$ one easily finds that all three matrix elements are of the type ${ }_{1}\left\langle\left|P_{12} P_{23}\right|\right\rangle_{1}$. The expressions given in [12] and [3] for $P$ are in fact evaluated there with the help of ${ }_{1}\left\langle\left|P_{12} P_{23}\right|\right\rangle_{1}$ and an originally occurring phase factor $(-)^{l^{\prime}+s^{\prime}+t^{\prime}}$ has been replaced by $(-1)$, since there only antisymmetric two-body states have been used. Redoing that replacement one finds the desired matrix element from the

one of $P$ given in [12] and [3] by multiplying it with $\frac{1}{2}(-)^{l^{\prime}+s^{\prime}+t^{\prime}+1}$. For the states without iso-spin one has just to drop the iso-spin factor.

Out of the many various forms to evaluate permutation operators [20] we need here three of them. The recoupling between the quasi two-body force quantities requires

$$
{ }_{1}\left\langle p q \alpha_{J}\left|P_{12} P_{23}\right| p^{\prime} q^{\prime} \alpha_{J}^{\prime}\right\rangle_{1}=\frac{1}{2}(-)^{l^{\prime}+s^{\prime}+1} \int_{-1}^{+1} d x \frac{\delta(p-\pi)}{p^{l+2}} \frac{\delta\left(p^{\prime}-\pi^{\prime}\right)}{p^{\prime l^{\prime}+2}} G_{\alpha_{J} \alpha_{J}^{\prime}}\left(q q^{\prime} x\right)
$$

with

$$
\begin{aligned}
\pi & =\sqrt{\frac{1}{4} q^{2}+q^{2}+q q^{\prime} x} \\
\pi^{\prime} & =\sqrt{q^{2}+\frac{1}{4} q^{2}+q q^{\prime} x}
\end{aligned}
$$

and

$$
G_{\alpha_{J} \alpha_{J}^{\prime}}\left(q q^{\prime} x\right)=\sum_{k} P_{k}(x) \sum_{l_{1}+l_{2}=l} \sum_{l_{1}^{\prime}+l_{2}^{\prime}=l^{\prime}} q^{l_{2}+l_{2}^{\prime}} q^{\prime l_{1}+l_{1}^{\prime}} g_{\alpha_{J} \alpha_{J}^{\prime}}^{k l_{1} l_{2} l_{1}^{\prime} l_{2}^{\prime}}
$$

The purely geometrical quantity $g_{\alpha_{J} \alpha_{J}^{\prime}}^{k l_{1} l_{2} l_{1}^{\prime} l_{2}^{\prime}}$ has to be taken now without iso-spin:

$$
\begin{aligned}
g_{\alpha_{J} \alpha_{J}^{\prime}}^{k l_{1} l_{2} l_{1}^{\prime} l_{2}^{\prime}} & =-\sqrt{\hat{l} \hat{s} \hat{j} \hat{\lambda} \hat{I} \hat{l}^{\prime} \hat{s}^{\prime} \hat{j}^{\prime} \hat{\lambda}^{\prime} \hat{I}^{\prime}} \\
& \times \sum_{L S} \hat{L} \hat{S}\left\{\begin{array}{ccc}
\frac{1}{2} & \frac{1}{2} & s \\
\frac{1}{2} & S & s^{\prime}
\end{array}\right\}\left\{\begin{array}{ccc}
l & s & j \\
\lambda & \frac{1}{2} & I \\
L & S & J
\end{array}\right\}\left\{\begin{array}{ccc}
l^{\prime} & s^{\prime} & j^{\prime} \\
\lambda^{\prime} & \frac{1}{2} & I^{\prime} \\
L & S & J
\end{array}\right\}
\end{aligned}
$$




$$
\begin{aligned}
& \times \hat{k}\left(\frac{1}{2}\right)^{l_{2}+l_{1}^{\prime}} \sqrt{\frac{(2 l+1) !}{\left(2 l_{1}\right) !\left(2 l_{2}\right) !} \sqrt{\frac{\left(2 l^{\prime}+1\right) !}{\left(2 l_{1}^{\prime}\right) !\left(2 l_{2}^{\prime}\right) !}}} \\
& \times \sum_{f f^{\prime}}\left\{\begin{array}{lll}
l_{1} & l_{2} & l \\
\lambda & L & f
\end{array}\right\}\left\{\begin{array}{ccc}
l_{2}^{\prime} & l_{1}^{\prime} & l^{\prime} \\
\lambda^{\prime} & L & f^{\prime}
\end{array}\right\} C\left(l_{2} 0 \lambda 0, f 0\right) C\left(l_{1}^{\prime} 0 \lambda^{\prime} 0, f^{\prime} 0\right) \\
& \times\left\{\begin{array}{ccc}
f & l_{1} & L \\
f^{\prime} & l_{2}^{\prime} & k
\end{array}\right\} C\left(k 0 l_{1} 0, f^{\prime} 0\right) C\left(k 0 l_{2}^{\prime} 0, f 0\right)
\end{aligned}
$$

The very left recoupling coefficient has to be taken in the form where the $\delta$-functions act both to the right:

$$
{ }_{1}\left\langle p q \alpha\left|P_{12} P_{23}\right| p^{\prime} q^{\prime} \alpha^{\prime}\right\rangle_{1}=\frac{1}{2}(-)^{l^{\prime}+s^{\prime}+t^{\prime}+1} \int_{-1}^{+1} d x \frac{\delta\left(p^{\prime}-\tilde{\pi}\right)}{p^{l^{\prime}+2}} \frac{\delta\left(q^{\prime}-\tilde{\chi}\right)}{q^{\prime \lambda^{\prime}+2}} \tilde{G}_{\alpha \alpha^{\prime}}(p q x)
$$

with

$$
\begin{aligned}
& \tilde{\pi}=\sqrt{\frac{1}{4} p^{2}+\frac{9}{16} q^{2}+\frac{3}{4} p q x} \\
& \tilde{\chi}=\sqrt{p^{2}+\frac{1}{4} q^{2}-p q x}
\end{aligned}
$$

and $\tilde{G}_{\alpha \alpha^{\prime}}(p q x)$ can be taken for instance from [3].

Finally the recoupling coefficient from the states of type 1 to 3 require a form where the $\delta$-functions act both to the left:

$$
\left\langle p q \alpha\left|P_{12} P_{23}\right| p^{\prime} q^{\prime} \alpha^{\prime}\right\rangle=\frac{1}{2}(-)^{l^{\prime}+s^{\prime}+t^{\prime}+1} \int_{-1}^{+1} d x \frac{\delta(p-\tilde{\tilde{\pi}})}{p^{l+2}} \frac{\delta(q-\tilde{\tilde{\chi}})}{q^{\lambda+2}} \tilde{G}_{\alpha^{\prime} \alpha}\left(p^{\prime} q^{\prime} x\right)
$$

with

$$
\begin{aligned}
& \tilde{\tilde{\pi}}=\sqrt{\frac{1}{4} p^{\prime 2}+\frac{9}{16} q^{\prime 2}-\frac{3}{4} p^{\prime} q^{\prime} x} \\
& \tilde{\tilde{\chi}}=\sqrt{p^{\prime 2}+\frac{1}{4} q^{\prime 2}+p^{\prime} q^{\prime} x}
\end{aligned}
$$

In eq. (A7) we need the same quantity $\tilde{G}$ as in eq. (A5), but with interchanged arguments.

\section{APPENDIX B: CUBIC HERMITIAN SPLINES}

In appendix $\mathrm{A}$ we encountered two-fold interpolations. They have to be performed for very many channels and should be as optimal as possible. We found that our usual 
basis splines [14] are not efficient enough to perform these two-fold interpolations within a reasonable time. Therefore we need a more efficient interpolation algorithm.

Such an algorithm can be found by using cubic hermitian splines. Although cubic hermitian splines are well known in the literature (see for example [21]), we will give here a short introduction in order to explain our way to use them.

Consider a function $f(x)$ given at certain grid points $\mathrm{x}_{i}, i=1, \ldots, n$. Let $x$ be positioned in the interval $\left[\mathrm{x}_{i}, \mathrm{x}_{i+1}\right]$. For the sake of simpler notation we call the end points $\mathrm{x}_{i} \equiv x_{1}$ and $\mathrm{x}_{i+1} \equiv x_{2}$. Then one defines an unique cubic polynomial $f_{i}(x)$ by the following constraints:

$$
\begin{aligned}
& f_{i}\left(x_{1}\right)=f\left(x_{1}\right) \\
& f_{i}\left(x_{2}\right)=f\left(x_{2}\right) \\
& f_{i}^{\prime}\left(x_{1}\right)=f^{\prime}\left(x_{1}\right) \\
& f_{i}^{\prime}\left(x_{2}\right)=f^{\prime}\left(x_{2}\right)
\end{aligned}
$$

Therefore these interpolating functions $f_{i}(x)$ and their derivatives $f_{i}^{\prime}(x)$ are continuous at the grid points $\mathrm{x}_{i}$. They are given by

$$
f_{i}(x)=f\left(x_{1}\right) \phi_{1}(x)+f\left(x_{2}\right) \phi_{2}(x)+f^{\prime}\left(x_{1}\right) \phi_{3}(x)+f^{\prime}\left(x_{2}\right) \phi_{4}(x)
$$

in terms of the spline functions

$$
\begin{aligned}
& \phi_{1}(x)=\left(\frac{\left(x_{2}-x\right)^{2}}{\left(x_{2}-x_{1}\right)^{3}}\left(\left(x_{2}-x_{1}\right)+2\left(x-x_{1}\right)\right)\right) \\
& \phi_{2}(x)=\left(\frac{\left(x_{1}-x\right)^{2}}{\left(x_{2}-x_{1}\right)^{3}}\left(\left(x_{2}-x_{1}\right)+2\left(x_{2}-x\right)\right)\right) \\
& \phi_{3}(x)=\frac{\left(x-x_{1}\right)\left(x_{2}-x\right)^{2}}{\left(x_{2}-x_{1}\right)^{2}} \\
& \phi_{4}(x)=\frac{\left(x-x_{1}\right)^{2}\left(x-x_{2}\right)}{\left(x_{2}-x_{1}\right)^{2}}
\end{aligned}
$$

We approximate the derivatives $f^{\prime}\left(x_{1}\right)$ and $f^{\prime}\left(x_{2}\right)$ with the help of a quadratic polynomial, which is uniquely defined by the function values at the grid point and its two neighbours. Calling $\mathrm{x}_{i-1}=x_{0}$ and $\mathrm{x}_{i+1}=x_{3}$ we get 


$$
\begin{aligned}
f^{\prime}\left(x_{1}\right) & \approx f\left(x_{2}\right) \frac{x_{1}-x_{0}}{x_{2}-x_{1}} \frac{1}{x_{2}-x_{0}}-f\left(x_{0}\right) \frac{x_{2}-x_{1}}{x_{1}-x_{0}} \frac{1}{x_{2}-x_{0}} \\
& +f\left(x_{1}\right)\left(\frac{x_{2}-x_{1}}{x_{1}-x_{0}}-\frac{x_{1}-x_{0}}{x_{2}-x_{1}}\right) \frac{1}{x_{2}-x_{0}} \\
f^{\prime}\left(x_{2}\right) & \approx f\left(x_{3}\right) \frac{x_{2}-x_{1}}{x_{3}-x_{2}} \frac{1}{x_{3}-x_{1}}-f\left(x_{1}\right) \frac{x_{3}-x_{2}}{x_{2}-x_{1}} \frac{1}{x_{3}-x_{1}} \\
& +f\left(x_{2}\right)\left(\frac{x_{3}-x_{2}}{x_{2}-x_{1}}-\frac{x_{2}-x_{1}}{x_{3}-x_{2}}\right) \frac{1}{x_{3}-x_{1}}
\end{aligned}
$$

At the end points $x_{1}$ and $x_{n}$ we define the quadratic polynomial by $f\left(\mathrm{x}_{1}\right), f\left(\mathrm{x}_{2}\right)$ and $f\left(\mathrm{x}_{3}\right)$ and by $f\left(\mathrm{x}_{n-2}\right), f\left(\mathrm{x}_{n-1}\right)$ and $f\left(\mathrm{x}_{n}\right)$, respectively. This is achieved by putting $x_{0}=\mathrm{x}_{3}$ in the first case and $x_{3}=\mathrm{x}_{n-2}$ in the second case.

Insertion of eq. (B4) into eq. (B2) yields

$$
f_{i}(x)=\sum_{j=0}^{3} S_{j}(x) f\left(x_{j}\right)
$$

for the interpolating function in the $i$-th interval of the grid points. Thereby we are lead to the modified spline functions

$$
\begin{aligned}
& S_{0}(x)=-\phi_{3}(x) \frac{x_{2}-x_{1}}{x_{1}-x_{0}} \frac{1}{x_{2}-x_{0}} \\
& S_{1}(x)=\phi_{1}(x)+\phi_{3}(x)\left(\frac{x_{2}-x_{1}}{x_{1}-x_{0}}-\frac{x_{1}-x_{0}}{x_{2}-x_{1}}\right) \frac{1}{x_{2}-x_{0}}-\phi_{4}(x) \frac{x_{3}-x_{2}}{x_{2}-x_{1}} \frac{1}{x_{3}-x_{1}} \\
& S_{2}(x)=\phi_{2}(x)+\phi_{3}(x) \frac{x_{1}-x_{0}}{x_{2}-x_{1}} \frac{1}{x_{2}-x_{0}}+\phi_{4}(x)\left(\frac{x_{3}-x_{2}}{x_{2}-x_{1}}-\frac{x_{2}-x_{1}}{x_{3}-x_{2}}\right) \frac{1}{x_{3}-x_{1}} \\
& S_{3}(x)=\phi_{4}(x) \frac{x_{2}-x_{1}}{x_{3}-x_{2}} \frac{1}{x_{3}-x_{1}}
\end{aligned}
$$

Eq. (B5) is very well suited for the numerical usage. The modified spline functions $S_{j}(x)$ are independent of the function values and depend only on the grid points and the actual position $x$. Therefore they can be prepared beforehand. This is very important if one has to interpolate very many functions given at the same grid points as we have to do in our 3NF code.

The form of eq. (B5) is the same as the one found in [14 for basis splines. The difference is, that the sum for the basis splines runs over the whole grid, whereas the sum for the hermitian splines in eq. (B5) has only four terms related to the four grid points nearest to the interpolation point $x$. (Basis splines are global splines, whereas hermitian splines 
are local.) Assuming a grid of typically 20 points the one-dimensional interpolation using hermitian splines needs only $\frac{1}{5}$ operations compared to basis splines. For a two-dimensional interpolation the number of operations is reduced by a factor of $\frac{1}{25}$.

For the two-dimensional interpolation one has to make a bi-cubic ansatz for the interpolating functions $f_{i j}(x, y)$. To define a bi-cubic function uniquely we need 16 constrains, which we choose as

$$
\begin{aligned}
f_{i j}\left(x_{1}, y_{1}\right) & =f\left(x_{1}, y_{1}\right) \\
\left.\frac{\partial f_{i j}\left(x, y_{1}\right)}{\partial x}\right|_{x=x_{1}} & =\left.\frac{\partial f\left(x, y_{1}\right)}{\partial x}\right|_{x=x_{1}} \\
\left.\frac{\partial f_{i j}\left(x_{1}, y\right)}{\partial y}\right|_{y=y_{1}} & =\left.\frac{\partial f\left(x_{1}, y\right)}{\partial y}\right|_{y=y_{1}} \\
\left.\frac{\partial^{2} f_{i j}(x, y)}{\partial x \partial y}\right|_{x=x_{1}, y=y_{1}} & =\left.\frac{\partial^{2} f(x, y)}{\partial x \partial y}\right|_{x=x_{1}, y=y_{1}}
\end{aligned}
$$

and identical expressions for the other three points $\left(x_{1}, y_{2}\right),\left(x_{2}, y_{1}\right)$, and $\left(x_{2}, y_{2}\right)$, respectively. Hereby the four grid points $\left(x_{1}, y_{1}\right),\left(x_{1}, y_{2}\right),\left(x_{2}, y_{1}\right)$ and $\left(x_{2}, y_{2}\right)$ are nearest neighbours to the interpolation point $(x, y)$ in the $x y$-plane.

The partial derivatives are approximated as in the one-dimensional case. The second derivative is estimated by a bi-quadratic polynomial, which is uniquely given by the function value at the specific grid point and the function values of the eight surrounding grid points.

Following these steps one yields

$$
f_{i j}(x, y)=\sum_{k=0}^{3} \sum_{l=0}^{3} S_{k l}^{(2)}(x, y) f\left(x_{k}, y_{l}\right)
$$

for the interpolating function. It is a fairly easy exercise to show that the two-dimensional spline functions $S_{k l}^{(2)}(x, y)$ are simply given by

$$
S_{k l}^{(2)}(x, y)=S_{k}(x) S_{l}(y)
$$

Analogical equations hold for interpolations in more than two dimensions.

To our experience one- and two-dimensional interpolations using hermitian splines are at least of the same accuracy as interpolations based on basis splines. 


\section{REFERENCES}

[1] Y. Wu, S. Ishikawa, T. Sasakawa, Few-Body Systems 15 (1993) 145

[2] W. Glöckle, H. Kamada, H. Witała, D. Hüber, J. Golak, K. Miyagawa, S. Ishikawa, Few-Body systems Suppl. 8 (1995) 9

[3] W. Glöckle, H. Witała, D. Hüber, H. Kamada, J. Golak, Phys. Rep. 274 (1996) 107

[4] S. Rosati, M. Viviani, A. Kievsky, Few-Body Systems Suppl. 8 (1995) 21

[5] H. Witała, D. Hüber, W. Glöckle, Few-Body Systems 16 (1994) 165

[6] W. Glöckle, H. Witała, H. Kamada, D. Hüber, J. Golak, AIP Conference Proceedings 334 on Few Body Problems in Physics, Williamsburg 1994, ed. F. Gross, AIP Press 1995, p. 45

[7] H. Patberg, R. Grossmann, G. Nitzsche, L. Sydow, S. Vohl, H. Paetz gen. Schieck, J. Golak, H. Witała, W. Glöckle, D. Hüber, Phys. Rev. C53 (1996) 1497

[8] M. Allet, K. Bodek, J. Golak, W. Glöckle, W. Hajdas, D. Hüber, L. Jarczyk, H. Kamada, St. Kistryn, J. Lang, R. Müller, O. Naviliat-Cuncic, J. Smyrski, J. Sromicki, A. Strzałkowski, H. Witała, J. Zejma, Phys. Lett. B376 (1996) 255

[9] S. A. Coon, W. Glöckle, Phys. Rev. C23 (1981) 1790

[10] S. A. Coon et al, Nucl. Phys. A317 (1979) 242

[11] C. R. Chen, G. L. Payne, J. L. Friar, B. F. Gibson, Phys. Rev. C33 (1986) 1740

[12] D. Hüber, H. Witała, W. Glöckle, Few-Body Systems 14 (1993) 171

[13] W. Glöckle, The Quantum Mechanical Few-Body Problem, Springer-Verlag, Berlin Heidelberg New York Tokyo 1983

[14] W. Glöckle, G. Hasberg, A. R. Neghabian, Z. Phys. A305 (1982) 217

[15] D. Hüber, PhD Thesis, Bochum 1993, unpublished 
[16] A. Stadler, W. Glöckle, P. U. Sauer, Phys. Rev. C44 (1991) 2319

[17] D. Hüber, H. Kamada, H. Witała, W. Glöckle, submitted for publication

[18] R. Machleidt, F. Sammarruca, Y. Song, Phys. Rev. C53 (1996) R1483

[19] R. B. Wiringa, R. A. Smith, T. L. Ainsworth, Phys. Rev. C29 (1984) 1207

[20] D. Hüber, H. Kamada, H. Witała, W. Glöckle, Few-Body Systems 16 (1994) 165

[21] H. Späth, Eindimensionale Spline-Interpolations-Algorithmen, R. Oldenbourg Verlag, München Wien 1990 


\section{TABLES}

\begin{tabular}{c|r|r|r}
\hline \hline$j_{\text {max }}$ & $E_{b}^{N N}$ & $E_{b}^{(1)}$ & $E_{b}^{(2)}$ \\
\hline 2 & 7.58 & 9.34 & 9.51 \\
3 & 7.67 & 9.27 & 9.25 \\
4 & 7.68 & 9.34 & 9.36 \\
5 & 7.69 & $9.32(9)$ & $9.32(4)$ \\
\hline \hline
\end{tabular}

TABLE I. Triton binding energies calculated using the AV14 NN force alone $\left(E_{b}^{N N}\right)$ and together with the TM $3 \mathrm{NF}\left(\Lambda=5.8 m_{\pi}\right)$. For explanation of $E_{b}^{(1)}$ and $E_{b}^{(2)}$ see text. 


\section{FIGURES}

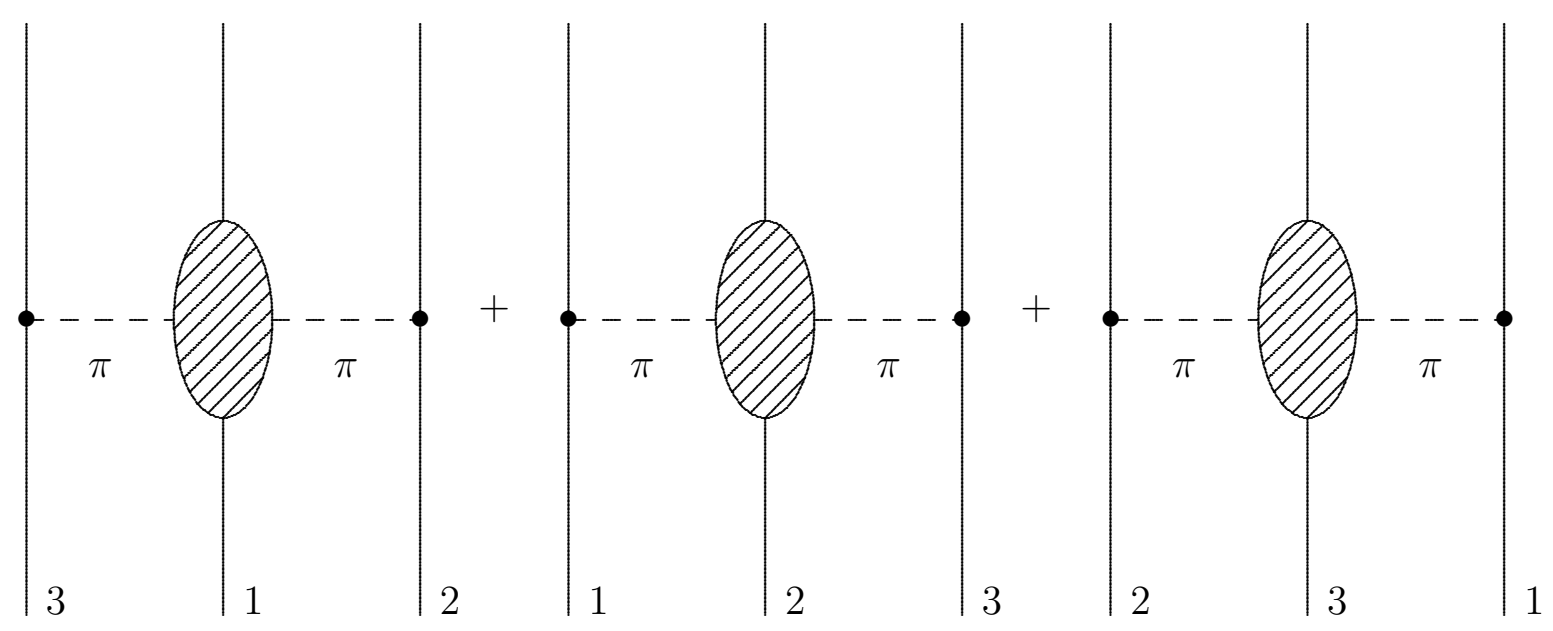

FIG. 1 . The $2 \pi$-exchange $3 \mathrm{NF}$.

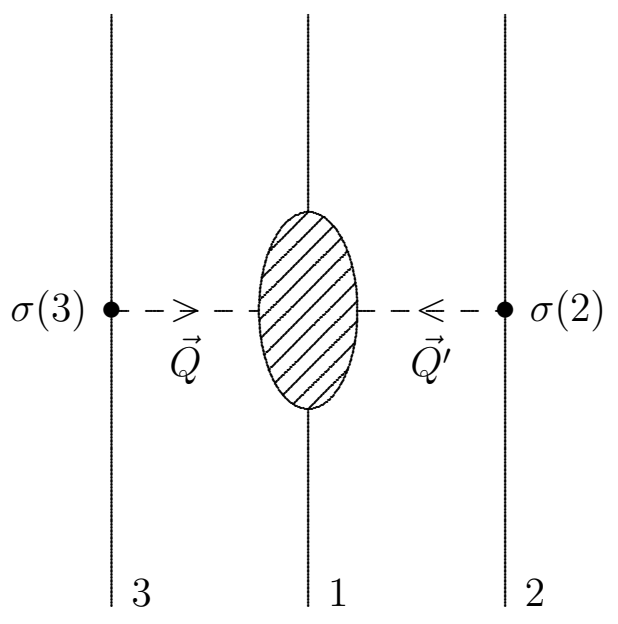

FIG. 2. The part $V_{4}^{(1)}$ of the $2 \pi$-exchange $3 \mathrm{NF}$. 

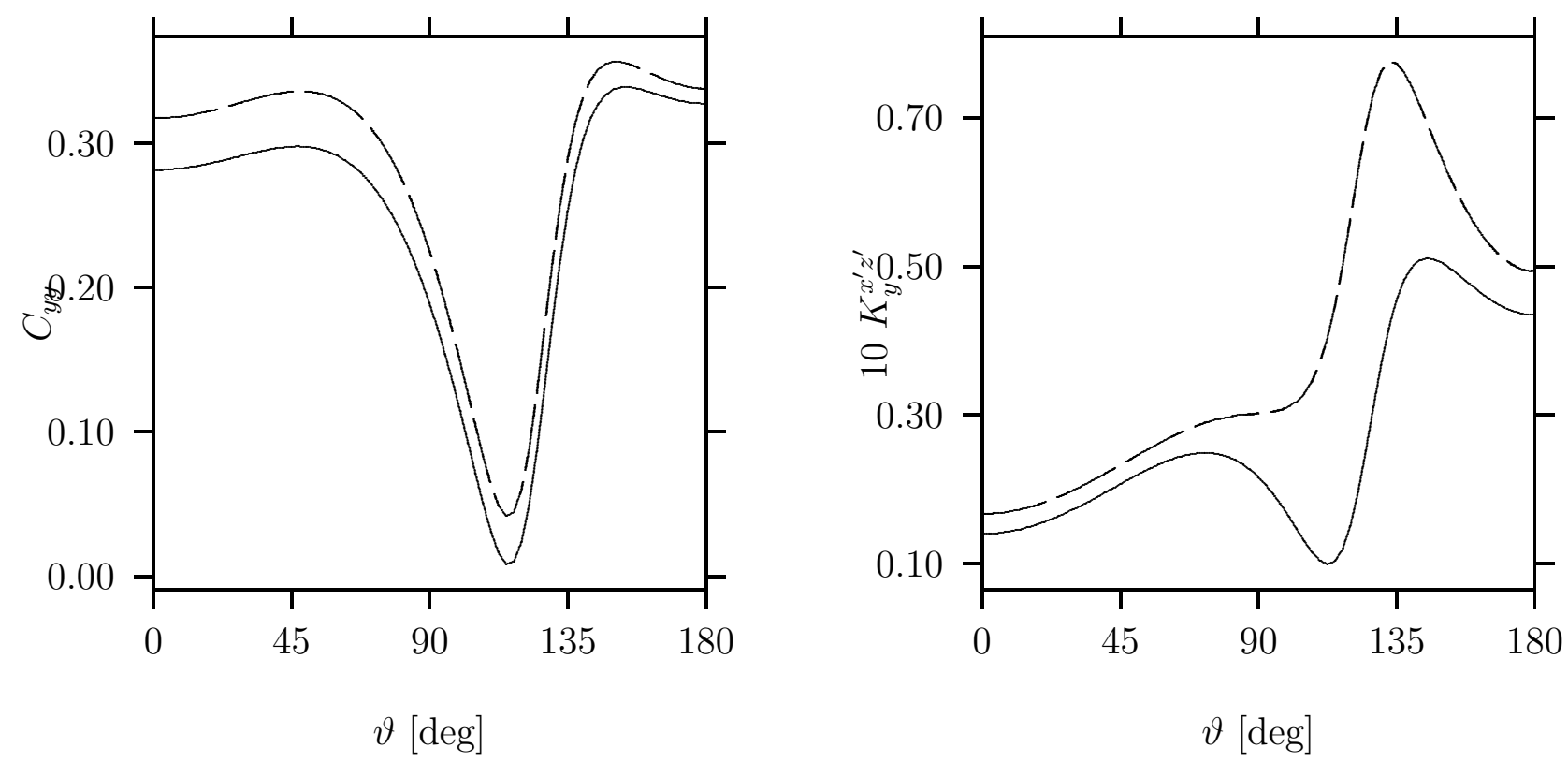

FIG. 3. The spin correlation coefficient $C_{y y}$ and the vector to tensor spin transfer coefficient $K_{y}^{x^{\prime} z^{\prime}}$ for elastic scattering at $E_{l a b}=10.3 \mathrm{MeV}$. The solid line is the result without $3 \mathrm{NF}$, the overlapping long and short dashed lines are the results with 3NF using the old and new PWD, respectively.
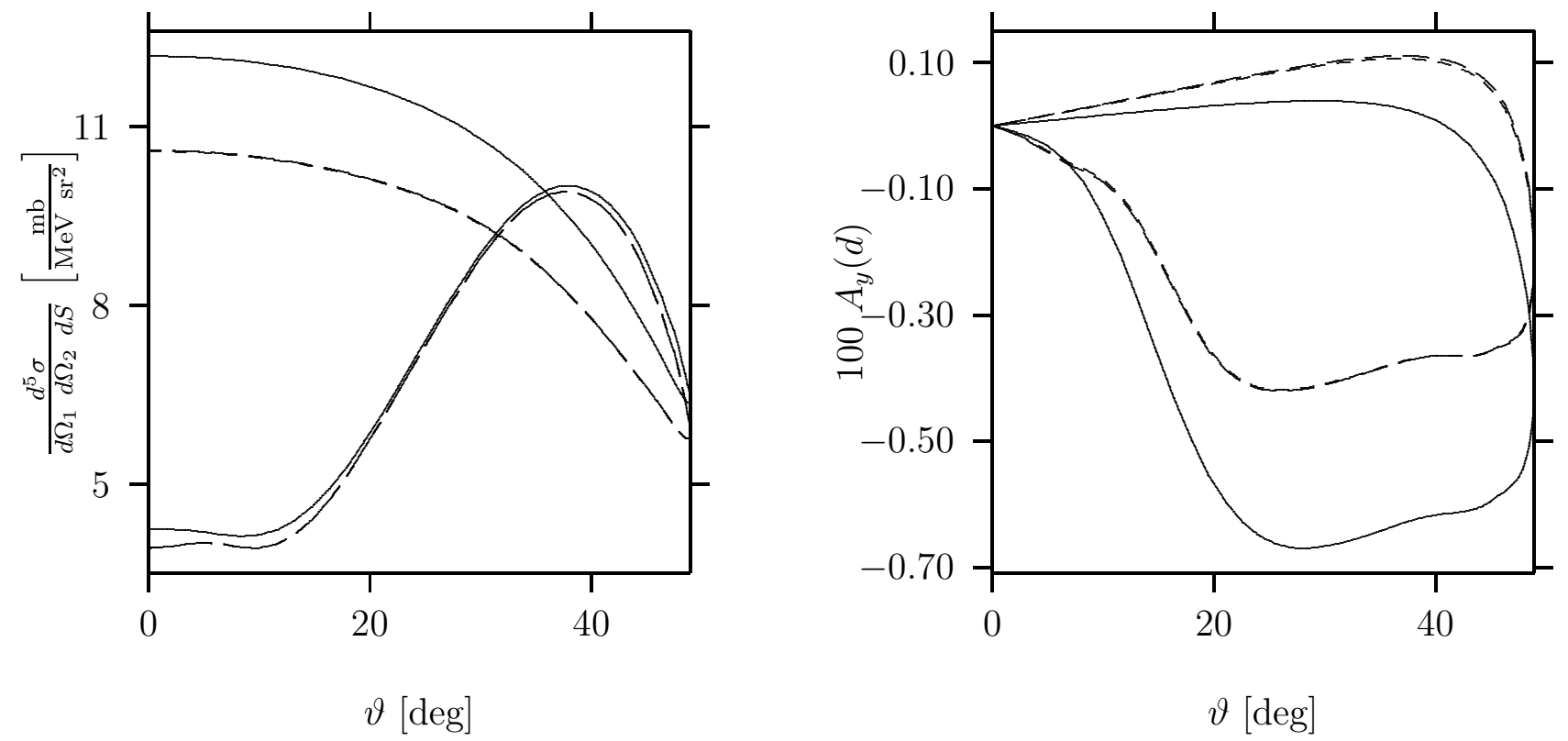

FIG. 4. The differential cross section and the deuteron analyzing power $A_{y}$ under np QFS conditions for the breakup process at $E_{l a b}=10.3 \mathrm{MeV}$ as functions of the scattering angle of one nucleon. Description as in Fig. 3. 